\title{
EFFICIENT BAYESIAN INFERENCE OF GENERAL GAUSSIAN MODELS ON LARGE PHYLOGENETIC TREES
}

\author{
By Paul Bastide ${ }^{1,2}$, LAm Si Tung Ho ${ }^{3}$, Guy Baele ${ }^{2}$, Philippe Lemey ${ }^{2}$ and MarC \\ A. SUCHARD ${ }^{4}$ \\ ${ }^{1}$ IMAG, CNRS, Université de Montpellier, paul.bastide@ umontpellier.fr \\ ${ }^{2}$ Department of Microbiology, Immunology and Transplantation, Rega Institute, KU Leuven, guy.baele@kuleuven.be; \\ philippe.lemey@kuleuven.be \\ ${ }^{3}$ Department of Mathematics and Statistics, Dalhousie University, lam.ho@dal.ca \\ ${ }^{4}$ Departments of Biostatistics, Biomathematics, and Human Genetics, University of California, Los Angeles, \\ msuchard@ucla.edu
}

\begin{abstract}
Phylogenetic comparative methods correct for shared evolutionary history among a set of non-independent organisms by modeling sample traits as arising from a diffusion process along the branches of a possibly unknown history. To incorporate such uncertainty, we present a scalable Bayesian inference framework under a general Gaussian trait evolution model that exploits Hamiltonian Monte Carlo (HMC). HMC enables efficient sampling of the constrained model parameters and takes advantage of the tree structure for fast likelihood and gradient computations, yielding algorithmic complexity linear in the number of observations. This approach encompasses a wide family of stochastic processes, including the general Ornstein-Uhlenbeck (OU) process, with possible missing data and measurement errors. We implement inference tools for a biologically relevant subset of all these models into the BEAST phylogenetic software package and develop model comparison through marginal likelihood estimation. We apply our approach to study the morphological evolution in the superfamilly of Musteloidea (including weasels and allies) as well as the heritability of HIV virulence. This second problem furnishes a new measure of evolutionary heritability that demonstrates its utility through a targeted simulation study.
\end{abstract}

\section{Introduction.}

1.1. Motivation. The evolutionary history of organisms shapes the distribution of their observed characteristics (Felsenstein, 1985). To account for correlation induced by this shared history, phylogenetic comparative methods (PCMs) have been developed for the analysis of quantitative traits (see e.g. Pennell and Harmon, 2013, for a review). These methods can be applied to a wide range of organisms and traits, to answer a large spectrum of biological questions on various evolutionary time frames, ranging from decades or even years in virology (Dudas et al., 2017) to millions of years in evolutionary biology (Aristide et al., 2016). Modern studies in PCMs routinely include a growing number of taxa (e.g. more than fifteen hundred in Blanquart et al. 2017), with possibly a large number of missing data for the multivariate measurements of continuous traits (e.g. almost $25 \%$ in Schnitzler et al. 2017), and with intricate phylogenetic and temporal structure. The underlying biological processes at play often have complex dynamics, and are only measured imperfectly, with a variable amount of noise.

1.2. Model. PCMs posit a continuous-valued stochastic process running on the branches of a phylogenetic tree that gives rise to trait values, possibly measured with noise, at the tree tips for observed organisms.

Keywords and phrases: Statistical Phylogenetics, Phylodynamics, Ornstein-Uhlenbeck Process, Bayesian Inference, Hamiltonian Monte Carlo, Model Selection, BBAST, Heritability, HIV, Musteloidea, Total Evidence 
Phylogenetic Tree. The phylogenetic tree represents the evolutionary relationship among the organisms studied. We assume, without loss of generality, that the tree is calibrated in time, so that branch lengths represent actual time. For organisms evolving rapidly, such as viruses, the observations at the $n$ tips of the tree are not necessarily contemporaneous (see Figure 1, left). We denote by $m$ the total number of internal and external nodes in the tree ( $m=2 n-1$ if the tree is binary).

Stochastic Process on the Tree. We assume that a continuous trait evolves over time according to a stochastic process. When a speciation event occurs in the tree, the process is split into two conditionally independent processes with the same distribution (see Figure 1). Only the values of the process at the tips of the tree are potentially observed. This model enforces a phylogenetic correlation structure on the observations, as shown below.
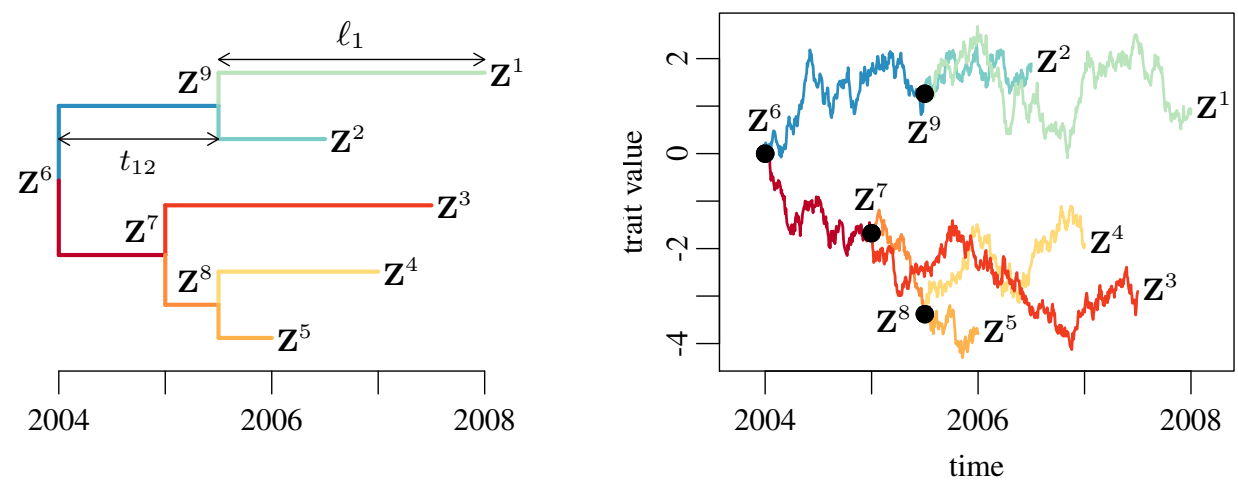

FIG 1. Realization of a univariate BM process (right) with variance $\sigma^{2}=0.05$ and mean root value $\mu=0$ on a timed phylogenetic tree (left). Observations span 3 years $\left(2006-2008\right.$, tips $\mathbf{Z}^{1}$ to $\left.\mathbf{Z}^{5}\right) . t_{12}$ is the time of shared evolution between tips 1 and $2 . \ell_{1}$ is the length of the branch ending at tip 1.

Brownian Motion (BM). The simplest stochastic process (Cavalli-Sforza and Edwards, 1967; Felsenstein, 1985) assumes that a multivariate trait $\mathbf{Z}_{t}$ of dimension $p$ evolves in time $t$ following a Brownian motion $\mathbf{B}_{t}$ with variance $\mathbf{R}$, such that $\mathrm{d} \mathbf{Z}_{t}=\mathbf{R}^{1 / 2} \mathrm{~d} \mathbf{B}_{t}$ for all $t \geq 0$ and at the tree root $\mathbf{Z}^{r} \sim \mathcal{N}(\boldsymbol{\mu}, \boldsymbol{\Gamma})$. Under this process, the covariance between trait $k$ at node $i$ and trait $l$ at node $j(1 \leq k, l \leq p$ and $1 \leq i, j \leq m)$ is the product of (i) the covariance $R_{k l}$ between traits $k$ and $l$ and (ii) the shared evolutionary time $t_{i j}$ between species $i$ and $j$, i.e. the time from the root to their most recent common ancestor (see Figure 1), plus the contribution of the root itself: $\operatorname{Cov}\left[Z_{k}^{i} ; Z_{l}^{j}\right]=t_{i j} R_{k l}+\Gamma_{k l}$ (see e.g. Clavel, Escarguel and Merceron, 2015). A constant drift $\nu$ can be added to the mean value of the trait (Gill et al., 2016), in which case the expectation of trait $k$ at species $i$ at time $t_{i}$ is $\mathbb{E}\left[Z_{k}^{i}\right]=t_{i} \nu$.

Ornstein-Uhlenbeck $(\mathrm{OU})$. The OU process was proposed as a model for traits evolving under stabilizing selection (Hansen, 1997) and has become widely used across evolutionary biology (see e.g. Cooper et al., 2016, and references therein). The OU process generalizes BM by adding a deterministic call-back term to a given value $\boldsymbol{\beta}$ that is interpreted as the optimal value of the trait of a species in a given environment: $\mathrm{d} \mathbf{Z}_{t}=-\mathbf{A}\left(\mathbf{Z}_{t}-\boldsymbol{\beta}\right) \mathrm{d} t+\mathbf{R}^{1 / 2} \mathrm{~d} \mathbf{B}_{t}$ for all $t \geq 0$. Matrix $\mathbf{A}$ is the "selection strength" that is constrained to have positive real parts of its eigenvalues and controls the dynamics of the pull toward the optimum. The covariance between two multivariate traits at two nodes can be explicitly formulated (Bartoszek et al., 2012; Clavel, Escarguel and Merceron, 2015), and, compared to the BM where the variance increases linearly in time, is bounded by the stationary variance $\mathbf{V}$ of the process. 
Observation Model and Individual Variation. The processes described above are meant to capture the evolutionary dynamics of traits across organisms. However, various other sources of variation may contribute to the observed data, such as measurement error, independent environmental variation, or intra-specific variation (see e.g. Hadfield and Nakagawa, 2010, for a review). As outlined in the next section, we include these biological phenomena in our model as an extra layer, that links the realization of the process at the tips $\mathbf{Z}^{i}$ to the actual measurements $\mathbf{Y}^{i}$ through a Gaussian observation model.

1.3. Scope of the article. In this work, we propose a general and efficient Bayesian framework to rigorously analyze this broad class of evolutionary models.

State of the Art. Since their introduction in the seminal article of Felsenstein (1985), PCMs have undergone extensive development, resulting in increasingly realistic models. We limit references here to those that specifically relate to the inference problem in a general setting and refer to e.g. Harmon (2019) for a recent and more comprehensive overview of these models. Clavel, Escarguel and Merceron (2015) provide a comprehensive maximum-likelihood framework to fit a wide range of models, using explicit estimators that can in some cases be computationally prohibitive. Pybus et al. (2012), Freckleton (2012), Mitov et al. (2019) describe and implement likelihood computation algorithms that are linear in the number of observations in a framework similar to the one described here. This algorithm is exploited in Mitov, Bartoszek and Stadler (2019) to conduct maximum-likelihood inference. From a Bayesian perspective, Pybus et al. (2012) and Cybis et al. (2015) describe a standard Markov chain Monte Carlo (MCMC) inference framework for BM, while Hassler et al. (2020) extend it to include measurement errors with possible missing values, and Fisher et al. (2020) to use an efficient Hamiltonian Monte Carlo (HMC) sampler on the rates of a relaxed random walk (Lemey et al., 2010). Other more general models have been developed, including: non Gaussian models (using e.g. Levy processes, see Landis, Schraiber and Liang 2013; Duchen et al. 2017, or general Fokker - Plank equations, see Boucher et al. 2018); models where the trait explicitly impacts the tree (with BiSSE and related methods, see Maddison, Midford and Otto 2007; Fitzjohn, Maddison and Otto 2009; Fitzjohn 2010; Goldberg, Lancaster and Ree 2011; Fitzjohn 2012); models where species interact with each other (through mutualism or competition, see Nuismer and Harmon 2015; Manceau, Lambert and Morlon 2016; Drury et al. 2016; Bartoszek et al. 2017; Drury et al. 2018; Aristide and Morlon 2019, or through migration Bartoszek 2017; Duchen et al. 2020a); models with varying and heritable intraspecific variance (Kostikova et al., 2016); or models in high-dimensional trait settings (using pseudo or penalized likelihood, see Goolsby 2016; Clavel, Aristide and Morlon 2019). These models are however outside of the scope of the present work, that focuses on the general Gaussian model as presented in the next section (see Definition 1).

Outline. We complement recent advances in computing the likelihood under a general class of Gaussian models with an algorithm to analytically evaluate its gradient with respect to any of the parameters in linear time in the number of observations. This general class includes the OU process, as well as measurement error and missing data. We exploit this algorithm to develop an efficient Bayesian inference framework that relies on the use of an HMC sampler and allows for model selection through marginal likelihood estimation. We implement this framework in the BEAST phylogenetic software package (Suchard et al., 2018) for a subclass of models, namely the OU process with a diagonal selection strength matrix A, that are of particular interest for the biological problems we study here. In Section 2, we present the general likelihood and new gradient computation algorithm (with details in Appendices A and B). In Section 3, we develop the Bayesian statistical inference framework (with details in Appendix C). Finally, in Section 4, we illustrate the method on two recently published biological datasets as well as on simulations. 
2. Efficient Gradient Computations. In this section, we show how the likelihood and its gradient with respect to all the parameters in a general trait evolutionary model can be computed in linear time in the number of tips of a rooted phylogenetic tree.

2.1. Statistical Model. Conditioning on a tree $\mathcal{T}$, we define the following general Gaussian model of trait evolution:

DEFINITION 1 (General Gaussian Model of Trait Evolution). Let $\mathcal{T}$ be a rooted phylogenetic tree with $n$ tips and $m$ internal and external nodes. At each node $j, 1 \leq j \leq m$, define a latent variable $\mathbf{Z}^{j}$, and for each observation $i, 1 \leq i \leq n$, a measure $\mathbf{Y}^{i}$, both of dimension $p$. The general Gaussian model of trait evolution on $\mathcal{T}$ is then defined in a hierarchical way as follows:

$$
\begin{aligned}
\mathbf{Z}^{r} & \sim \mathcal{N}(\boldsymbol{\mu}, \boldsymbol{\Gamma}) & & \text { root; } \\
\mathbf{Z}^{j} \mid \mathbf{Z}^{\mathrm{pa}(j)} & \sim \mathcal{N}\left(\mathbf{q}_{j} \mathbf{Z}^{\mathrm{pa}(j)}+\mathbf{d}_{j}, \mathbf{\Sigma}_{j}\right) & & \text { propagation; } \\
\mathbf{Y}^{i} \mid \mathbf{Z}^{\mathrm{pa}(i)} & \sim \mathcal{N}\left(\mathbf{Z}^{\mathrm{pa}(i)}, \mathbf{S}_{i}\right) & & \text { observation; }
\end{aligned}
$$

where $\mathrm{pa}(j)$ and $\mathrm{pa}(i)$ denote, respectively, the unique parent node of node $j$ or latent tip associated with observation $i ; \boldsymbol{\mu}$ and $\boldsymbol{\Gamma}$ are the expectation and variance of the root variable $\mathbf{Z}^{r}$; for any node $j, \mathbf{q}_{j}, \mathbf{d}_{j}$ and $\boldsymbol{\Sigma}_{j}$ are, respectively, the actualization, drift, and variance associated with the branch going from $\operatorname{pa}(j)$ to $j$; and for any observation $i, \mathbf{S}_{i}$ is the variance associated with it. We further denote by $\mathbf{Y}=\left(\mathbf{Y}^{1}, \cdots, \mathbf{Y}^{n}\right)^{T}$ and $\mathbf{Z}=\left(\mathbf{Z}^{1}, \cdots, \mathbf{Z}^{m}\right)^{T}$ the matrices of observed and latent trait variables, and by $\mathbf{X}=\left(\mathbf{Y}^{T}, \mathbf{Z}^{T}\right)^{T}$ the complete dataset. Furthermore, we assume that $\boldsymbol{\Gamma}, \boldsymbol{\Sigma}_{j}$, and $\mathbf{S}_{i}$ are positive definite for any node $j$ and observation $i$.

This allows entertaining a highly generic framework that encompasses various evolutionary scenarios, as underlined in the following three paragraphs.

Stochastic Process Propagation. Equation (2.2) describes the stochastic process that governs the evolution of the latent trait. It is similar to the $\mathcal{G}_{\text {Lnv }}$ model described in Mitov et al. (2019), or the generic formulation used in Bastide et al. (2018). Both the BM and OU models can be cast into this framework, by setting:

$$
\begin{array}{llll}
\mathbf{q}_{j}=\mathbf{I}_{p}, & \mathbf{d}_{j}=\ell_{j} \boldsymbol{\nu}, & \boldsymbol{\Sigma}_{j}=\ell_{j} \mathbf{R} & (\mathrm{BM}) ; \\
\mathbf{q}_{j}=e^{-\mathbf{A} \ell_{j}}, & \mathbf{d}_{j}=\left(\mathbf{I}_{p}-e^{-\mathbf{A} \ell_{j}}\right) \boldsymbol{\beta}, & \boldsymbol{\Sigma}_{j}=\mathbf{V}-e^{-\mathbf{A} \ell_{j}} \mathbf{V} e^{-\mathbf{A}^{T} \ell_{j}} &
\end{array}
$$

where, as defined in the introduction, $\boldsymbol{\nu}$ and $\mathbf{R}$ are the constant drift and variance of a simple $\mathrm{BM}$, and $\mathbf{A}, \boldsymbol{\beta}$ and $\mathbf{V}$ are the selection strength, optimal values and stationary variances of an OU. We refer to Mitov et al. (2019) for more details concerning other models that can be described within this framework, including processes with shifts or jumps.

Observation Model. Equation (2.3) describes the observation model. The variance term $\mathbf{S}_{i}$ can have multiple significations, from a simple measurement error, to a "meta-analysis" effect (see Hadfield and Nakagawa, 2010 for a review on observation errors), or an "intra-specific" variance (see e.g. Goolsby, Bruggeman and Ané, 2017). In the simple case where the same observation error is assumed for all measures $i, 1 \leq i \leq n$, this term reduces to $\mathbf{S}_{i}=\mathbf{S}$. Note that, from a methodological point of view, Equation (2.3) can be considered as a particular case of Equation (2.2). 
Phylogenetic Factor and Repetitions. We note that one may posit that $\mathbf{Z}^{i}$ is of lower dimension $q<p$ and link to $\mathbf{Y}^{i}$ through a linear combination via a latent factor model (Tolkoff et al., 2018). It would also be straightforward to include several measurements associated with a single tip. For clarity, we omit these details in the main text, and refer to Appendix A for the derivations in this general framework.

2.2. Likelihood Computations. The general model of Definition 1 is Gaussian, and it is hence possible to write out the marginal distribution $p(\mathbf{Y} \mid \boldsymbol{\theta})$ of the measures $\mathbf{Y}$ given the parameters $\boldsymbol{\theta}$ for some specific models (see e.g. Clavel, Escarguel and Merceron, 2015 for such formulations in the multivariate OU case). However, this computation generally requires the inversion of a tree-induced variance matrix, of dimension $n \times n$. This is inefficient (worse than quadratic in $n$, see e.g. Raz, 2003), and is ill-suited for handling large phylogenetic trees that now frequently confront practitioners (see e.g. Jetz et al., 2012; Blanquart et al., 2017) or when the tree itself is random (Pybus et al., 2012). To alleviate this issue, it is possible to write an efficient pruning-style algorithm that is linear in the number $n$ of organisms.

Pruning-Style Algorithm. Felsenstein (1973) introduced the pruning algorithm into phylogenetics to compute the likelihood of a simple BM. It draws from classical Gaussian conditional propagation ideas such as the Kalman filter or other "forward-backward" algorithms (see e.g. Rabiner, 1989 for a review). Variants of this algorithm have been flowering in the literature, sometimes under different names. A non-exhaustive list of references for the BM case includes Hadfield and Nakagawa (2010), Pybus et al. (2012), Fitzjohn (2012) (Gaussian Elimination Method), Freckleton (2012), Lartillot (2014) (Phylogenetic Kalman Filter) and Cybis et al. (2015). Goolsby (2016) and Hassler et al. (2020) have recently proposed adaptations to handle missing data. Finally, an extension of the algorithm to the general case as presented in Definition 1 (with missing data) was proposed by Bastide et al. (2018), and also more recently by Mitov et al. (2019).

Implementation in BEAST. All these methods allow computing the likelihood in linear time in $n$. As is frequently the case for PCMs, many have been implemented in various independent software packages, each likely with a specific use in mind (with the notable exception of the R Core Team, 2019 package PCMbase, see Mitov et al., 2019). While this explains at least partly the numerous references to the algorithm, it limits the use of several implementations beyond the specific models they consider. Bayesian Evolutionary Analysis by Sampling Trees (BEAST, Suchard et al., 2018) is a widely used, well established and versatile phylogenetic software package. It encompasses a great variety of molecular sequence modeling tools, making it possible to conduct a coherent joint inference of both the timed phylogenetic tree and of the properties of the stochastic process, without the need to resort to a two-step analysis as is usually the case in previous methodologies (see Section 3.1 for more details). We implemented the general algorithm presented in Bastide et al. (2018) in this unified framework (with improvements, see next paragraph), allowing for its seamless integration with the realistic analyses permitted by the software.

Efficiency and Numerical Robustness. When sampling the parameters in a wide region of the space, as is typically done in a Bayesian analysis (see Section 3), numerical robustness is particularly important, as small divergences due to possibly ill-conditioned matrices can accumulate over the tree traversal, and lead to diverging results. We tackled this issue using two independent developments. First, we reduced the number of operations actually performed during the traversal of the tree thanks to a careful analysis of the iteration steps, making the algorithm both more efficient and more robust. Second, we increased the numerical robustness by using a dedicated linear algebra library (the Efficient Java Matrix library, Abeles, 2016) to conduct the computations. Combined with the use of a Moore-Penrose pseudo inverse, 
this made our handling of the singular or near-singular matrices induced by the presence of missing data (see Bastide et al., 2018, Hassler et al., 2020) more numerically stable. These developments are presented in detail in Appendix A.1.

2.3. Gradient Computation. When performing statistical inference, either in a maximum likelihood or a Bayesian framework, having access to the gradient of the likelihood at relatively cheap computational cost facilitates faster and more accurate algorithms (see Section 3). In this section, we present a novel algorithm to compute the gradient of the likelihood with respect to any parameter in the general setting presented in Definition 1 . The algorithm relies on two main ingredients: (1) as in Fisher et al. (2020), we express the derivative of the likelihood as the conditional expectation of a given function of the latent traits $\mathbf{Z}^{j}$ at the internal nodes $j$, conditional on the observed traits $\mathbf{Y}$; and (2) we use a pre-order algorithm inspired from the "downward" phase in Bastide et al. (2018) to compute this expectation in a linear time in $n$.

Gradient as a Conditional Expectation. We rely here on Fisher's identity (Cappé, Moulines and Rydén, 2005) that links the gradient of the $\log$-likelihood $\log p(\mathbf{Y} \mid \boldsymbol{\theta})$ of the observed variables to the conditional expectation of the completed $\log$-likelihood $\log p(\mathbf{Z}, \mathbf{Y} \mid \boldsymbol{\theta})$ :

Proposition 1 (Fisher's Identity; Cappé, Moulines and Rydén, 2005). Under broad assumptions, that are verified for Gaussian densities, the following identity holds (Equation 10.12 in Cappé, Moulines and Rydén, 2005):

$$
\nabla_{\boldsymbol{\theta}}[\log p(\mathbf{Y} \mid \boldsymbol{\theta})]=\mathbb{E}\left[\nabla_{\boldsymbol{\theta}}\left[\log p\left(\mathbf{X}_{s}, \mathbf{Y} \mid \boldsymbol{\theta}\right)\right] \mid \mathbf{Y}\right],
$$

where $\mathbf{X}_{s}$ represents any subset taken from the complete data.

Applying this identity, we obtain the gradient of the likelihood with respect to any parameter $\boldsymbol{\theta}_{j}=\left(\mathbf{q}_{j}, \mathbf{d}_{j}, \boldsymbol{\Sigma}_{j}\right)$ or $\boldsymbol{\theta}_{i}=\mathbf{S}_{i}$ of the model:

PROPOSITION 2 (Gradient with respect to Branch Parameters). Under the general model of Definition 1, for any observation or node $k(1 \leq k \leq n+m)$, the following identity holds:

$$
\begin{aligned}
\nabla_{\boldsymbol{\theta}_{k}}\left[\log p\left(\mathbf{Y} \mid \boldsymbol{\theta}_{k}\right)\right] & =\frac{\partial \mathbf{n}_{k}^{T}}{\partial \boldsymbol{\theta}_{k}} \cdot \mathbf{Q}_{k}\left(\mathbf{M}_{k}-\mathbf{n}_{k}\right) \\
& +\frac{\partial \operatorname{vech}\left(\mathbf{Q}_{k}\right)^{T}}{\partial \boldsymbol{\theta}_{k}} \cdot \frac{1}{2} \operatorname{vech}\left(\mathbf{Q}_{k}^{-1}-\left(\mathbf{M}_{k}-\mathbf{n}_{k}\right)\left(\mathbf{M}_{k}-\mathbf{n}_{k}\right)^{T}-\mathbf{V}_{k}\right)
\end{aligned}
$$

with vech the symmetric vectorization operation (Magnus and Neudecker, 1986); and where $\mathbf{n}_{k}, \mathbf{M}_{k}, \mathbf{Q}_{k}^{-1}$ and $\mathbf{V}_{k}$ are parameters, representing the expectations and variances of two Gaussian densities (see Equations A.15 and A.23 in Appendix A.2), that can be computed in one pre-order traversal of the tree.

PROOF OF PROPOSITION 2. Let $k$ be an observation or node index with associated trait variable $\mathbf{X}^{k}(1 \leq k \leq n+m)$. As in Fisher et al. (2020), we decompose the observations $\mathbf{Y}$ as $\mathbf{Y}=\left(\mathbf{Y}_{\lfloor k\rfloor}, \mathbf{Y}_{\lceil k\rceil}\right)$, where $\mathbf{Y}_{\lfloor k\rfloor}$ denotes the observations that are "below" node $k$, i.e. that have $k$ as an ancestor, and $\mathbf{Y}_{\lceil k\rceil}$ denotes the observations that are "above" node $k$, i.e. that do not have $k$ as an ancestor. The tree conditional structure then induces the decomposition:

$$
p\left(\mathbf{X}^{k}, \mathbf{Y}_{\lfloor k\rfloor}, \mathbf{Y}_{\lceil k\rceil} \mid \boldsymbol{\theta}_{k}\right)=p\left(\mathbf{Y}_{\lfloor k\rfloor} \mid \mathbf{X}^{k}\right) p\left(\mathbf{X}^{k} \mid \mathbf{Y}_{\lceil k\rceil}, \boldsymbol{\theta}_{k}\right) p\left(\mathbf{Y}_{\lceil k\rceil}\right),
$$


where only the middle term depends on parameters $\boldsymbol{\theta}_{k}$ associated with the branch ending at node $k$. Applying Proposition 1 with $\mathbf{X}_{s}=\mathbf{X}^{k}$, we get that:

$$
\nabla_{\boldsymbol{\theta}_{k}}\left[\log p\left(\mathbf{Y} \mid \boldsymbol{\theta}_{k}\right)\right]=\mathbb{E}\left[\nabla_{\boldsymbol{\theta}_{k}}\left[\log p\left(\mathbf{X}^{k} \mid \mathbf{Y}_{\lceil k\rceil}, \boldsymbol{\theta}_{k}\right)\right] \mid \mathbf{Y}\right] .
$$

Using the pre-order formulas presented in Appendix A.2.1 (Equation A.15), we can see that $\mathbf{X}^{k} \mid \mathbf{Y}_{\lceil k\rceil}$ is normally distributed, with expectation $\mathbf{n}_{k}$ and precision matrix $\mathbf{Q}_{k}$. Applying standard derivation formulas to a log Gaussian density (see e.g. Magnus and Neudecker, 1986), we have:

$$
\begin{aligned}
\nabla_{\boldsymbol{\theta}_{k}}\left[\log p\left(\mathbf{X}^{k} \mid \mathbf{Y}_{\lceil k\rceil}, \boldsymbol{\theta}_{k}\right)\right]=\frac{\partial \mathbf{n}_{k}^{T}}{\partial \boldsymbol{\theta}_{k}} \cdot \mathbf{Q}_{k}\left(\mathbf{X}^{k}-\mathbf{n}_{k}\right) \\
+\frac{\partial \operatorname{vech}\left(\mathbf{Q}_{k}\right)^{T}}{\partial \boldsymbol{\theta}_{k}} \cdot \frac{1}{2} \operatorname{vech}\left(\mathbf{Q}_{k}^{-1}-\left(\mathbf{X}^{k}-\mathbf{n}_{k}\right)\left(\mathbf{X}^{k}-\mathbf{n}_{k}\right)^{T}\right),
\end{aligned}
$$

From Appendix A.2.2 (Equation A.23), we know that $\mathbf{X}^{k} \mid \mathbf{Y}$ is normally distributed, with expectation $\mathbf{M}_{k}$ and variance $\mathbf{V}_{k}$. Equation (2.7) is then obtained by taking the conditional expectation of the above expression (2.8).

Chain Rule. In Equation (2.7), we express the gradient of the likelihood with respect to any branch parameter using only quantities that can be computed in two traversals of the tree, one post-order and one pre-order. This provides the basis for an algorithm to compute the gradient of the likelihood with respect to any parameter with a linear complexity in $n$. Indeed, one only needs to apply the derivation chain rule to: (1) obtain the gradient of the branch parameters $\mathbf{n}_{k}$ and $\mathbf{Q}_{k}$ with respect to the natural parameters of the process at hand; and (2) obtain the gradient of the likelihood with respect to parameters shared between several branches. We tackle task (1) using the pre-order formulas in Appendix B. Task (2) is a straightforward application of the chain rule over all the levels of the hierarchical model:

$$
\frac{\partial p(\mathbf{Y} \mid \boldsymbol{\theta}, \mathcal{T})^{T}}{\partial \boldsymbol{\theta}}=\sum_{k=1}^{n+m} \frac{\partial \boldsymbol{\theta}_{k}^{T}}{\partial \boldsymbol{\theta}} \nabla_{\boldsymbol{\theta}_{k}}[p(\mathbf{Y} \mid \boldsymbol{\theta}, \mathcal{T})] .
$$

Complexity. Appendix A implies that all the moments $\mathbf{n}_{k}, \mathbf{M}_{j}, \mathbf{Q}_{k}^{-1}$ and $\mathbf{V}_{k}(1 \leq k \leq$ $n+m$ ) appearing in Proposition 2 can be computed in linear time in the number of observations. Since formulas (2.7) and (2.9) only involve linear algebra operations in a space of the dimension of the parameters, the total complexity remains linear in $n$. In addition, we note that the sum in Equation (2.9) does not need to follow the tree order, as all the quantities are pre-computed, and hence can be parallelized easily, reducing the actual computation time.

3. Statistical Inference. In the previous section, we showed how both the likelihood and its gradient with respect to all the parameters of the models can be efficiently computed simultaneously. These quantities are the cornerstone of many statistical analyses, and allow for a wide range of analyses, from maximum likelihood to model selection (for examples in trait evolution, see e.g. Clavel, Aristide and Morlon, 2019). Here, taking advantage of the comprehensive Bayesian inference framework made available through the BEAST phylogenetics software package, we propose a new Bayesian approach that relies on the use of an efficient HMC sampler to perform both posterior inference and marginal likelihood estimation. 
3.1. Bayesian Phylogenetics and the Total Evidence Approach. The likelihood and gradient algorithms presented below work conditionally on a phylogenetic tree between sampled species being known without error. However, the tree is generally a summary statistic resulting from a complex statistical analysis, and is usually inferred from molecular sequences, which are the actual observed data. Many methods in the literature follow a two-step procedure and first infer the tree from sequence data to then proceed with analyzing the continuous traits, assuming that the phylogenetic tree is known and fixed (see e.g. Harmon, 2019 for a recent review of such methods). This approach suffers from two major drawbacks. First, it ignores the uncertainty in the reconstruction of the tree, which, given the difficulty of the task, can be substantial, and bias the subsequent analyses (see Felsenstein, 2004 for a review, and Bastide, 2017, Section 5.1, for an example in a specific case). Second, this approach does not allow for the complete use of the data available, as it ignores continuous traits for the tree reconstruction. Although, when present, sequence information tends to dominate over trait information (Baele et al., 2017), it is not always available for all sampled organisms. This is particularly true for ancient fossils, that might bear some continuous trait data, but, because of the rapid degradation of DNA molecules, can not be sequenced (Leonardi et al., 2016). We refer to Section 4.3 for an example of such a dataset, where the continuous trait constitutes the only source of information available to reconstruct the phylogenetic tree.

To overcome these limitations, we use a total evidence approach (Ronquist et al., 2012), that can analyze sequence and trait data jointly, using all the information available in a Bayesian analysis. Denote by $\mathbf{S}$ the sequence data (that might not be available for all the sampled species), and by $\phi$ all the parameters associated with the model of sequence evolution and the dating clock model, (see e.g. Felsenstein, 2004 for a review of such models). The goal of Bayesian phylogenetics is then to learn about the posterior: $p(\boldsymbol{\theta}, \mathcal{T}, \boldsymbol{\phi} \mid \mathbf{Y}, \mathbf{S})$. One crucial assumption that we make is that, conditionally on the phylogenetic tree $\mathcal{T}$, the evolution of continuous traits and the sequences are independent, such that:

$$
\begin{aligned}
p(\boldsymbol{\theta}, \mathcal{T}, \boldsymbol{\phi} \mid \mathbf{Y}, \mathbf{S}) & \propto p(\mathbf{Y}, \mathbf{S} \mid \boldsymbol{\theta}, \mathcal{T}, \boldsymbol{\phi}) p(\boldsymbol{\theta}, \mathcal{T}, \boldsymbol{\phi}) \\
& =p(\mathbf{Y} \mid \boldsymbol{\theta}, \mathcal{T}) p(\boldsymbol{\theta}) \times p(\mathbf{S} \mid \mathcal{T}, \boldsymbol{\phi}) p(\mathcal{T}, \boldsymbol{\phi}) .
\end{aligned}
$$

The term $p(\mathbf{S} \mid \mathcal{T}, \boldsymbol{\phi}) p(\mathcal{T}, \boldsymbol{\phi})$ has been the focus of an extensive literature, and benefits from efficient methods readily available in BEAST (Suchard et al., 2018). Thanks to the tools presented in the previous section, we focus here on $p(\mathbf{Y} \mid \boldsymbol{\theta}, \mathcal{T}) p(\boldsymbol{\theta})$ that deals with the study of the distribution of continuous traits among the population of species.

This conditional independence assumption, although limiting, is essential from a computational point of view. It has also proven to be useful and adequate to study a wide range of biological questions, and it is widely spread in the field of PCMs, applied to a fixed tree or in a total evidence approach (see e.g. Felsenstein, 2004; Harmon, 2019, for reviews). As mentioned in the Introduction (see Section 1.3), some attempts have been made to relax this assumption, with fixed trees or discrete characters (see e.g. Fitzjohn 2012; Müller, Rasmussen and Stadler 2017 and references therein). However, the computational burden associated to these methods currently limits their application to relatively small scale datasets.

3.2. Hamiltonian Monte Carlo. $\mathrm{HMC}$ is a powerful MCMC sampling technique, that exploits the geometrical properties of the density to be sampled through the use of Hamiltonian dynamics (Neal, 2011; Betancourt, 2017). It associates to a vector of parameters of interest $\boldsymbol{\theta}$, viewed as the position of a particle in a $d$-dimensional space, an auxiliary independent vector $\mathbf{p}$ of "momentum", that is typically chosen to be Gaussian: $\mathbf{p} \sim \mathcal{N}\left(\mathbf{0}_{d}, \mathbf{I}_{d}\right)$. The log joint distribution of the parameter $(\boldsymbol{\theta}, \mathbf{p})$ then represents the "total energy" $\mathrm{H}(\boldsymbol{\theta}, \mathbf{p})=\mathrm{U}(\boldsymbol{\theta})+\mathrm{K}(\mathbf{p})$ of the particle, with $\mathrm{U}(\boldsymbol{\theta})=-\log p(\boldsymbol{\theta} \mid \mathbf{Y}, \mathcal{T})$ the "potential energy" set to be equal to the 
posterior density of interest, and $K(\mathbf{p})=\mathbf{p}^{T} \mathbf{p} / 2$ the "kinetic energy". The total energy is then invariant to the Hamiltonian dynamics:

$$
\left\{\begin{array}{l}
\frac{\mathrm{d} \mathbf{p}}{\mathrm{d} t}=-\nabla_{\boldsymbol{\theta}} \mathrm{U}(\boldsymbol{\theta})=\nabla_{\boldsymbol{\theta}} \log p(\mathbf{Y} \mid \boldsymbol{\theta}, \mathcal{T})+\nabla_{\boldsymbol{\theta}} \log p(\boldsymbol{\theta}) \\
\frac{\mathrm{d} \boldsymbol{\theta}}{\mathrm{d} t}=+\nabla_{\mathbf{p}} \mathrm{K}(\mathbf{p})=\mathbf{p} .
\end{array}\right.
$$

The HMC sampling scheme exploits this property using proposals that approximately follow these dynamics, as discretized by an appropriate numerical scheme such as the leapfrog. This allows for a proposal that can have a small correlation with the current state, while still having a high probability of acceptation (Neal, 2011). Such an HMC sampler has already proven very successful in a phylogenetics context (Fisher et al., 2020; Ji et al., 2020). Our efficient and general algorithm for likelihood and gradient computation, presented in Section 2, makes it now applicable to the wide variety of models covered by Definition 1.

3.3. Confronting Constrained Natural Parameters. Some of the parameters of the models, such as the variance of a BM or an OU, live in constrained spaces with a non-trivial structure, that need to be sampled adequately. One standard way to deal with this structure (see e.g. Stan, 2017) is to map the constrained natural parameters $\boldsymbol{\theta}$ to a vector of independent, unconstrained parameters $\boldsymbol{\eta}$ through a smooth transformation $f$. The density in the new, unconstrained space is then linked to the density in the constrained space by a simple multiplication with the determinant of the Jacobian matrix of the transformation (see e.g. Le Gall, 2006), such that:

$$
\nabla_{\boldsymbol{\eta}} \log p(\boldsymbol{\eta})=\frac{\partial f^{-1}(\boldsymbol{\eta})^{T}}{\partial \boldsymbol{\eta}} \nabla_{\boldsymbol{\theta}} \log p\left(f^{-1}(\boldsymbol{\eta})\right)+\nabla_{\boldsymbol{\eta}} \log \left|\frac{\partial f^{-1}(\boldsymbol{\eta})^{T}}{\partial \boldsymbol{\eta}}\right| .
$$

This formula allows us to easily update the constrained parameters $\theta$ from movements in the unconstrained space of $\boldsymbol{\eta}$. We present the transformations and the associated priors used here in detail in Appendix C. In particular, we show that sampling the space of correlation matrices amounts to sampling vectors in the half-euclidean sphere, which provides an original and simple representation of the classical LKJ transformation (Lewandowski, Kurowicka and Joe, 2009).

3.4. Model Selection and Marginal Likelihood Estimation. We described above a general framework to efficiently infer the parameters of a wide class of evolutionary models. When analyzing a dataset, a question that naturally arises is the choice of the most suited model to interpret the evolutionary patterns in a specific problem.

Bayesian Model Selection. In a Bayesian setting, one natural way to compare a collection of models $\left(\mathcal{M}_{m}\right)_{1 \leq m \leq K}$ is to compute their marginal likelihoods $p\left(\mathbf{Y} \mid \mathcal{M}_{m}\right)$ (see e.g. Oaks et al. 2019 for an introduction in a phylogenetic context). The marginal likelihood, that integrates all the parameters against the prior, takes the model complexity into account by design, "penalizing" complex models, that otherwise mechanically have a higher likelihood. Marginal likelihoods allow computing Bayes factors, which have a natural comparison scale (Jeffreys, 1935; Kass and Raftery, 1995). However, because of the need to integrate over the potentially very large space of parameters, this quantity is typically hard to compute, and approximations are required. 
Generalized Stepping-Stone Sampling (GSS). In a phylogenetic context, the GSS approach (Fan et al., 2011) has been successfully used to approximate marginal likelihoods (Baele, Lemey and Suchard, 2016; Fourment et al., 2019). For a given model $\mathcal{M}$, it relies on the construction and sampling of a path between the unnormalized posterior and a "working" prior distribution $p_{0}(\boldsymbol{\theta} \mid \mathcal{M})$ :

$$
q_{\beta}(\boldsymbol{\theta})=[p(\mathbf{Y} \mid \boldsymbol{\theta}, \mathcal{T}, \mathcal{M}) p(\boldsymbol{\theta} \mid \mathcal{M})]^{\beta}\left[p_{0}(\boldsymbol{\theta} \mid \mathcal{M})\right]^{1-\beta} .
$$

When $\beta=1$, the path likelihood is proportional to the classical posterior sampled in a standard MCMC analysis, while when $\beta=0$, it reduces to the working prior $p_{0}(\boldsymbol{\theta} \mid \mathcal{M})$. This working prior is chosen to match the empirical moments from a sample of the posterior distribution, ensuring a less vague distribution that is closer to the posterior, hence inducing a more accurate approximation for a reduced computational effort than the standard stepping-stone sampling procedure (Xie et al., 2011; Fan et al., 2011). As in Baele, Lemey and Suchard (2016), we adopt a kernel density estimator (KDE) for each parameter, using a normal kernel, that is log-transformed for positive parameters (see e.g. Jones, Nguyen and McLachlan, 2018).

Sampling the path with HMC. The GSS estimation implies sampling from the path likelihood $q_{\beta}(\boldsymbol{\theta})$ for a sequence of $\beta$. Xie et al. (2011) and Baele, Lemey and Suchard (2016) show that choosing the path parameter as evenly spaced quantiles of a Beta distribution with shape 0.3 and scale 1.0 , which allows for sampling more intensely regions where $\beta$ is small, and hence where the path likelihood is changing the most rapidly, yields the best performance. This sampling is usually done through a standard MCMC procedure. Here, we use the efficient HMC approach presented in Section 3.2, which implies taking the gradient of the log path likelihood (3.2):

$$
\nabla_{\boldsymbol{\theta}} \log q_{\beta}(\boldsymbol{\theta})=\beta \nabla_{\boldsymbol{\theta}} \log [p(\mathbf{Y} \mid \boldsymbol{\theta}, \mathcal{T}, \mathcal{M}) p(\boldsymbol{\theta} \mid \mathcal{M})]+(1-\beta) \nabla_{\boldsymbol{\theta}} \log p_{0}(\boldsymbol{\theta} \mid \mathcal{M}) .
$$

This gradient involves a term proportional to the posterior that we already dealt with in the HMC inference, and the working distribution, that, as a product of independent KDE estimations, is straightforward to compute. This makes it possible to use the efficient HMC sampling scheme in the GSS marginal likelihood estimation framework already implemented and well established in BEAST (Baele, Lemey and Suchard, 2016; Fourment et al., 2019).

\section{Applications and Simulations.}

4.1. Assumptions and Practical Implementation. We showcase the usefulness of our inference framework using two recently published datasets, one in ecology, and one in virology. This led us to implement a subset from all the models made accessible by the method. Specifically, we limit the evolutionary model to the BM and the OU with diagonal strength of selection $\mathbf{A}$, and a shared residual variance for all the measures, possibly scaled by the tip heights $\left(\mathbf{S}_{i}=\mathbf{S}\right.$ or $\mathbf{S}_{i}=t_{i} \mathbf{S}$ for any observation $\left.i, 1 \leq i \leq n\right)$.

4.2. Phylogenetic Heritability. The concept of phylogenetic heritability has been defined in the field of PCMs to study the relative importance of the evolution and observation models in the total measured variance at the tip of the tree (Lynch, 1991; Housworth, Martins and Lynch, 2004). It is linked to the notion of phylogenetic signal (Pagel, 1999), and its use has recently received considerable attention in studying infection traits in the field of virology (Alizon et al., 2010; Leventhal and Bonhoeffer, 2016; Mitov and Stadler, 2018). We introduce here a general definition of the phylogenetic heritability that extends this notion to our general 
framework. It relies on the expectation of the population variances computed at the latent tip level (for the $\mathbf{Z}^{\text {tip }}=\left(\mathbf{Z}^{j}\right)_{1 \leq j \leq n}$ ), and at the observation level (for the $\mathbf{Y}=\left(\mathbf{Y}^{i}\right)_{1 \leq i \leq n}$ ):

$$
\begin{aligned}
\mathbf{V}\left(\mathbf{Z}^{\mathrm{tip}}\right) & =\mathbb{E}\left[\frac{1}{n}\left[\mathbf{Z}^{\mathrm{tip}}-\mathbb{E}_{\boldsymbol{\theta}}\left[\mathbf{Z}^{\mathrm{tip}}\right]\right]^{T}\left[\mathbf{Z}^{\mathrm{tip}}-\mathbb{E}_{\boldsymbol{\theta}}\left[\mathbf{Z}^{\mathrm{tip}}\right]\right]=\frac{1}{n} \sum_{j=1}^{n} \operatorname{Var}_{\boldsymbol{\theta}}\left[\mathbf{Z}^{j}\right],\right. \\
\mathbf{V}(\mathbf{Y}) & =\mathbb{E}\left[\frac{1}{n}\left[\mathbf{Y}-\mathbb{E}_{\boldsymbol{\theta}}[\mathbf{Y}]\right]^{T}\left[\mathbf{Y}-\mathbb{E}_{\boldsymbol{\theta}}[\mathbf{Y}]\right]\right]=\frac{1}{n} \sum_{i=1}^{n} \operatorname{Var}_{\boldsymbol{\theta}}\left[\mathbf{Y}^{i}\right],
\end{aligned}
$$

where the expectation and variance are taken following the process of evolution and observation defined in Definition 1 with parameters $\boldsymbol{\theta}$. These quantities have closed-form expressions for all the models considered here, see e.g. Clavel, Escarguel and Merceron (2015) for the general OU case. The "heritability matrix" $\mathbf{H}$ can then be defined as:

$$
H_{k l}=\frac{V_{k l}\left(\mathbf{Z}^{\mathrm{tip}}\right)}{\sqrt{V_{k k}(\mathbf{Y}) V_{l l}(\mathbf{Y})}} .
$$

In the case of a standard univariate trait on an ultrametric tree with only one observation per tip, this formula coincides with the classical definition found in the literature (see e.g. Mitov and Stadler, 2018).

Population versus Empirical Variance. In equation (4.3), we use the population variance, instead of the empirical one used for instance in Blanquart et al. (2017), Hassler et al. (2020). We argue in Appendix D that, when the process is not a simple BM, the population variance is more appropriate, as the empirical variance might be impaired by confounding inter-group effects if the tips are expected to have different means under the trait evolution model, which is for instance the case for an OU model on a non-ultrametric tree.

4.3. Morphological Evolution in the Musteloidea Superfamily. We illustrate the total evidence approach to study the evolution of some morphological features in the Musteloidea superfamilly (including weasels and allies).

\subsubsection{Dataset and Analyses.}

Dataset. We reanalyze the dataset published by Schnitzler et al. (2017), containing 81 taxa, including 4 fossils, and 2 outgroup species. Aligned sequence data for all 77 extant taxa are available (containing 22 nuclear and 5 mitochondrial genes). Three morphological traits are measured on 65 species, including fossils, with missing data for some taxa (see Figure 2). They are carnivorian ecometric traits, defined as meaningful ratios of osteological measurements, and denoted by R1-3. Note that our Bayesian framework can readily handle this heterogeneous dataset, and jointly analyse sequence and continuous traits with missing data on both.

Questions. We aim to address the following two questions. First, does total evidence (see Section 3.1) allow for better placement of the fossils? Given that we can take trait data into account while performing phylogenetic inference, we might expect that this extra information leads to better fossil placement estimates compared with Schnitzler et al. 2017, who only considered sequence data and use monophyly constraints. Second, which model of trait evolution is most suited to explain the observed trait distribution, and does this conclusion change when we include or set aside fossil data, as suggested by Schnitzler et al. (2017)? 


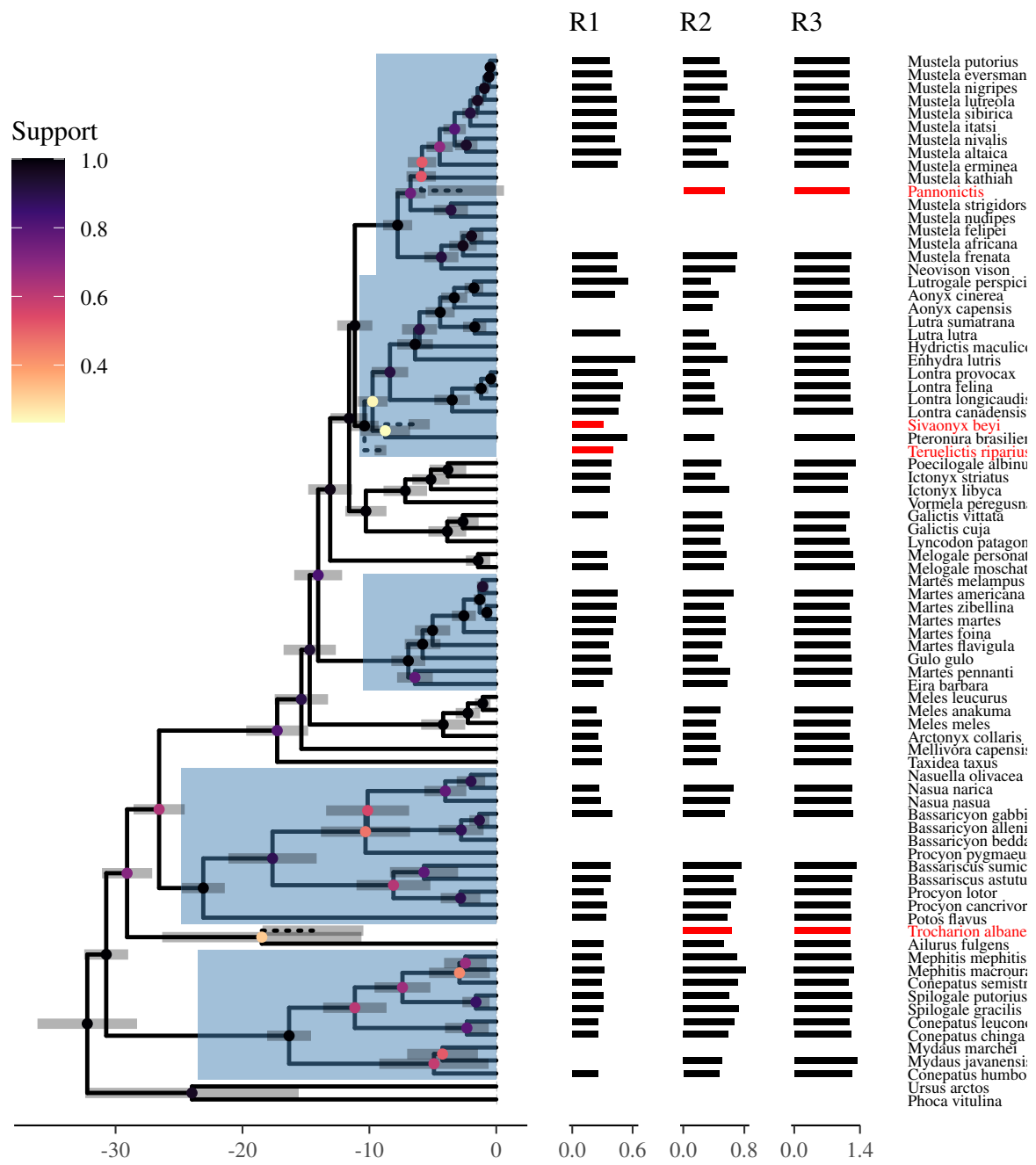

FIG 2. Maximum clade credibility tree with continuous traits plotted at the tips. The tree is reconstructed from both the genetic data and the trait data, using an OU model with a diagonal selection strength. The time scale for the tree is in million of years, with 0 indicating the present. Clades highlighted in blue are constrained to be monophyletic. Grey bars at nodes and fossils show the dating uncertainty. Node colors indicate the posterior support for each clade. Fossils are dotted, and highlighted in red.

Sequence Evolution and Dating. We use the same sequence evolution model as Schnitzler et al. (2017) for all 27 partitions of the dataset, with estimated base frequencies and site rate heterogeneity modeled using a discretized gamma distribution with 6 rate categories; an uncorrelated relaxed clock with an underlying gamma distribution; and an exponential growth coalescent tree prior (Hasegawa, Kishino and Yano, 1985; Tavaré, 1986; Yang, 1994; Drummond et al., 2006). Following Schnitzler et al. (2017), we constrain 5 clades to be monophyletic, and each fossil is a priori assigned to one of these clades, except for Trocharion albanense, which remains unconstrained (see Figure 2). We assume a normal prior on the time of the most recent common ancestor for each of those clades, with means as in Schnitzler et al. (2017), and standard deviation 1. We assume a uniform prior on fossil dates, with maximum ranges taken from Law, Slater and Mehta (2018) (Table S5), except for Teruelictis riparius, for which dates were extracted from the Paleobiology Database, relying on Salesa 
et al. (2013). Note that these assumptions differ slightly from Schnitzler et al. (2017), who provide insufficient information to reproduce their exact pipeline.

Total Evidence Phylogenetic Inference. We conduct phylogenetic inference using 3 different data integration scenarios: no model of trait evolution (i.e. the continuous traits are not used); a BM model; and an OU with diagonal selection strength. The latter two combine both sequence and trait evolution. We run each analysis for 100 million iterations, sample every 1000 steps, and discard the first $10 \%$ as burn-in. We specified vague priors on the parameters of the continuous processes while respecting biological constraints, as described in Appendix C. The maximum clade credibility (MCC) tree is used to represent the evolutionary history.

Fossil Placement Analysis. We assess the uncertainty of fossil placement using a method introduced by Klopfstein and Spasojevic (2019). Given a sample of trees from the posterior, as the backbone tree is well resolved (see below), the method amounts to computing, for each branch of the MCC tree, the frequency a given fossil attaches to that branch. We measure frequency vector concentration using entropy; a fossil that is well resolved will be distributed over a small number of branches with high frequency, and hence has a low entropy.

Model Comparison. As in Schnitzler et al. (2017), we also conduct several model comparisons, conditioning on a tree fixed to the MCC tree from one of the previous analyses, with or without fossil species. On each tree, we test several hypotheses about the nature of trait evolution, by comparing ( $\log$ ) marginal likelihood estimates for the various models. Models tested in this section are the BM and OU models, but also the "trend" model, that is a BM with an added homogeneous deterministic drift (Hansen and Martins, 1996; Gill et al., 2016). See Supplementary Figure S4 for the list of all hypotheses tested. We run each analysis for 100000 iterations, sample every 10 steps, and discard the first $10 \%$ as burn-in. 50 steps of 1000 iterations each are explored for the GSS estimation of the $(\log )$ marginal likelihood of each model.

Analysis and Representation of the Results. We use BEAST, TreeAnnotator and Tracer to conduct the analyses (Suchard et al., 2018; Rambaut et al., 2018). Trees are imported into R and plotted using treeio (Wang et al., 2019), tidytree and ggtree (Yu et al., 2017, 2018).

\subsubsection{Results.}

Phylogenetic Inference. Consistent with Schnitzler et al. (2017), we estimate a wellresolved backbone tree, with uncertainty mostly at the genus level, particularly in the Mephitidae and Procyonidae families (see Figure 2). Including trait information does not dramatically change the inferred relationships between extant species, confirming that molecular data are generally more informative than trait data (Baele et al., 2017).

Fossil Placement. Taking into account trait information reduces entropy scores for each of the fossils, with the OU model having the lowest entropy for $3 / 4$ of the fossils (see Table 1 and Supplementary Figure S3). Continuous trait measurements bear different amounts of information for each fossil, which leads to different entropy score behavior. The fossil Pannonictis is evenly distributed over all the branches of the clade (Mustelinae) where it is assigned. It has a high entropy that does not decrease much when traits are taken into account. This result is not surprising given that the traits vary little among all the species of this clade (see Figure 2), and hence yield little information with respect to fossil placement. Fossils Sivaonyx beyi and Teruelictis riparius are both assigned to the same clade (Lutrinae). Figure 2 illustrates that their R1 trait is relatively lower compared to other members of the clade. Taking this trait into account is thus informative, and entropy decreases, with the fossil estimated to lie at the root of the tree (see Supplementary Figure S3). Finally, the species 
Trocharion albanense is not assigned to any clade. Taking traits into account concentrates this fossil as a sister lineage either to Ailurus fulgens, or to the whole Mephitidae clade, which, in the assumed time range, have the most similar traits.

\begin{tabular}{lrrrr}
\hline & Pannonictis & Sivaonyx beyi & Teruelictis riparius & Trocharion albanense \\
\hline No Traits & 2.84 & 1.80 & 1.24 & 2.28 \\
BM & $\mathbf{2 . 8 1}$ & 1.22 & 1.22 & 2.01 \\
OU & 2.92 & $\mathbf{1 . 2 0}$ & $\mathbf{1 . 1 0}$ & $\mathbf{1 . 8 6}$ \\
\hline
\end{tabular}

Entropy of the fossil position for each fossil and inference method (over 1000 trees from the posterior). Entropy should decrease if the fossils are better resolved. Maximal entropy (no information) is 5.02. OU models appear to reduce entropy for three fossils out of four.

Model Comparisons. We find that the favored model, for all tested trees, with or without fossils, is a simple BM for the first trait (R1), and an OU with diagonal selection strength but full correlation for the two other traits (R2 and R3), with R1 evolving independently from R2 and R3 (see Supplementary Figure S4). The parameter estimates are consistent with those from Schnitzler et al. (2017) (see Supplementary Figure S5). Schnitzler et al. (2017) fitted the three traits independently and used a simple penalized likelihood approach (using the Akaike Information Criterion, Akaike 1974) to demonstrate that a "trend" model is favored to the simple BM model for R1 when fossils are included. In contrast, our method is robust to the addition of these fossils that, given the missing data, amounts to the addition of two data points in the analysis (see Figure 2). The selected model has a log Bayes factor of at least 1.5 compared to the second best fitting model in all the scenarios, providing "substantial evidence" (Kass and Raftery, 1995) against the simple BM model.

4.4. Virulence Heritability in Human Immunodeficiency Viruses (HIV). New challenges for PCMs have recently emerged in infectious disease research, more specifically on the extent to which virulence is a heritable trait in HIV. Here, we employ our new modeling framework to perform a fine-grained analysis to gain insight into this problem.

\subsubsection{Dataset and Analyses.}

Dataset. We revisit the most comprehensive dataset on HIV-1 heritability published in Blanquart et al. (2017) and further analysed in Hassler et al. (2020). We focus on subtype $\mathrm{B}$ and the measurements available for male subjects who have sex with men (MSM), which comprises a dataset of 1171 viral samples. Two traits associated with HIV virulence (Alizon et al., 2010; Blanquart et al., 2017) are measured for each sample: (i) the "gold standard viral load" (GSVL) that is a standardized measure of the viral load, taken on a single sample between 6 and 24 months after infection and before initiation of antiretroviral therapy; and (ii) the CD4 cell count slope decline (see Figure 3). A dated maximum likelihood phylogeny for this dataset has recently been presented by Hassler et al. (2020). Following a similar methodology, we use it as a fixed tree in our analyses, which focus on continuous trait model selection and heritability estimations.

Questions. Disease progression varies greatly among patients. Similar to other rapidly evolving human pathogens, it is challenging to determine to what extent this variance is due to the host or virulence of the viral genotype. The pioneering application of PCMs by Alizon et al. (2010) to estimate the heritability of HIV virulence using set-point viral load 

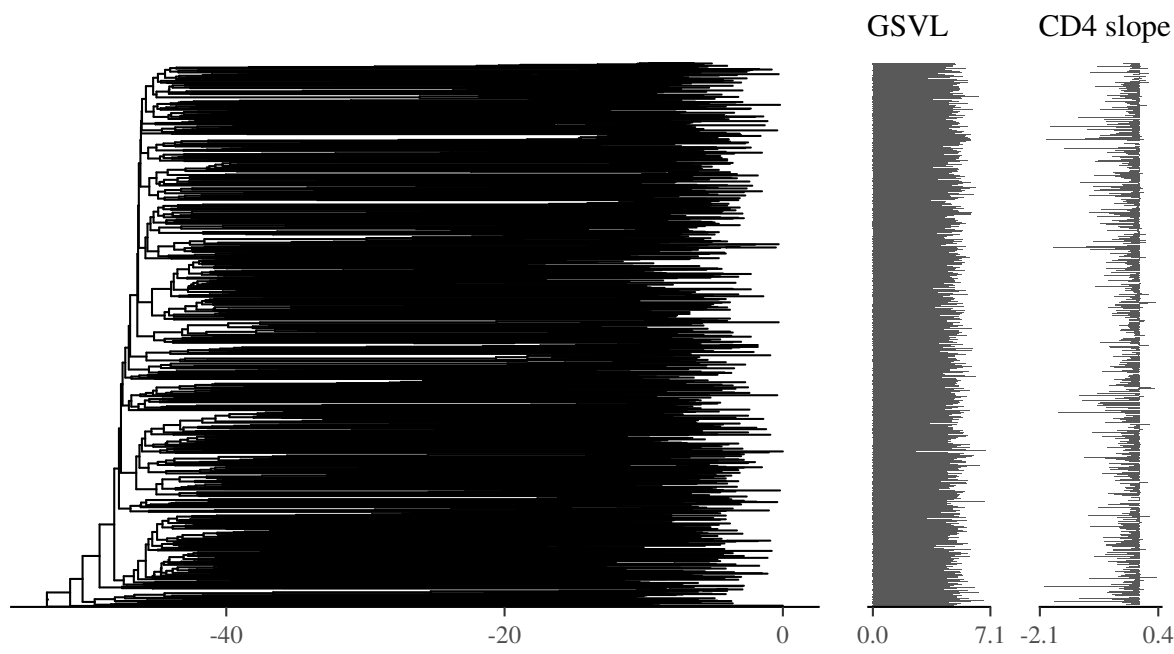

FIG 3. HIV-1 dataset from Blanquart et al. (2017) and Hassler et al. (2020). A maximum-likelihood tree estimate depicts the phylogenetic relationships for all HIV-1 subtype B viruses from MSM patients. The time scale is in calendar years (O being the present, putting the root at around 1960). The two traits are the GSVL and the CD4 count slope decrease.

( $\mathrm{spVL}$ ), has stimulated the generation of comprehensive data sets (Blanquart et al., 2017), but also led to a discussion concerning the underlying models (Mitov and Stadler, 2018; Bertels et al., 2018). Depending on the method and datasets used, the heritability of the spVL has been quite controversial (Leventhal and Bonhoeffer, 2016), with estimates ranging from about $50 \%$ (Alizon et al., 2010), to around 30\% (Vrancken et al., 2015) and to as low as about $6 \%$ (Hodcroft et al., 2014). We explore here the fit of several models of trait evolution and individual variation (Equations 2.2 and 2.3) to study their impact on heritability estimation and other parameters of interest. As the virus-host interactions are a major source of trait variation, we expect the individual variation layer to be particularly important in these models.

Trait Evolution Models. We use three different evolution models for the two traits on the tree: a multivariate $\mathrm{BM}$, a multivariate $\mathrm{OU}$ (with diagonal selection strength), and a mixed multivariate "OU-BM" model, that has an OU model on the GSVL, and a BM model on the CD4 slope, the two still being correlated. This last model illustrates the flexibility of our framework in model specification. It is motivated by the results presented in Blanquart et al. (2017), and by the data distribution (see Figure 3), with the CD4 slope being much more spread out than the GSVL (with respective quartile coefficients of dispersion of -0.56 and $0.09)$.

Individual Variation Models. One major driver of diversity for the two traits is the interaction of the virus with its host, that is independent from the viral phylogeny. This individual variation is captured through our observation model layer. We take this variation to be either identically distributed, or scaled by the tip heights, with or without trait correlation. Scaling the independent noise by the tip heights is an empirical model inspired from Pagel's $\lambda$ model (Pagel, 1999) and is well suited for an environmental contribution that increases linearly with sampling time (Leventhal and Bonhoeffer, 2016).

Model Comparison and Model Fit. As in the previous example using a fixed tree, and using the same computational tools, priors (see Appendix C), and chain settings, we estimate (log) marginal likelihoods for all the models under study. 


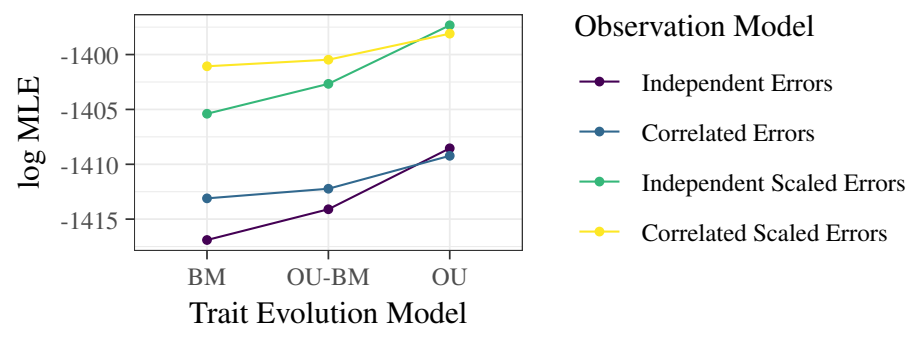

FIG 4. Log Marginal Likelihood Estimation (log MLE) for three different trait evolution models (BM on both traits, $O U$ on GSVL and BM on CD4, and OU on both traits) and four observation models, either independent or correlated, and scaled by tip heights or not. The best fitting model is the OU with independent scaled errors, with a log Bayes factor of 0.77 compared to the second best model, the more complex OU with correlated scaled errors.

\subsubsection{Results.}

Model Selection. The model favored according to the log marginal likelihood estimation is the OU model on both traits, with an independent, scaled observation matrix (see Figure 4). Compared to the OU-BM model with the same error structure, it has a log Bayes factor support of 3.14, indicating a strong support for the more complex OU model (Kass and Raftery, 1995). In general, the scaled error models appear to be much better supported than the non-scaled ones.

Heritability. Under the best fitted model, the heritability is estimated to be, respectively, 0.3 (95\% highest posterior density interval: [0.16, 0.45]) for the GSVL, and 0.36 (95\% HPDI $[0.15,0.65])$ for the CD4 slope. This is in line with the selection strength estimates, with a phylogenetic half-life (in percentage of the tree height) of 0.27 (95\% HPDI $[0.1,0.61]$ ) for the GSVL, and $0.12(95 \%$ HPDI $[0.05,0.26])$ for the CD4 slope. The CD4 slope has a higher selection strength, so that the phylogenetic model allows for more individual variation (see e.g. Bastide et al., 2018), and hence the heritability, which is the relative importance of this phylogenetic model in the total variation, is mechanically higher. See Appendix E.2 for a complete presentation of the results.

Discussion. The results presented above are surprising on two accounts. First, the heritability results under the best supported OU model are different from the ones reported in Blanquart et al. (2017), who find a heritability that is larger for the GSVL than for the CD4, with estimates of 0.31 (95\% HPDI $[0.15,0.43]$ ) for the GSVL, and 0.1 (95\% HPDI $[0.01,0.27])$ for the CD4 slope. We note however that the selection strength parameter in Blanquart et al. (2017) is poorly estimated, with a wide confidence interval that abuts against the lower and upper limits that were arbitrarily imposed: the half-life is estimated to 0.08 (95\% HPDI $[0.07,0.29])$ for the GSVL, and $7.3\left(95 \%\right.$ HPDI $\left.\left[0.0693,6.3 \times 10^{5}\right]\right)$ for the CD4 slope. In contrast, the priors we set, that reflect biologically reasonable assumptions, might help us regularize the estimation of this notoriously hard to infer parameter (see e.g. Uyeda and Harmon, 2014, Bastide, Mariadassou and Robin, 2017).

Second, the log MLE favors a model with an OU on the CD4 slope, while Blanquart et al. (2017) favor a BM on this trait, which seems more reasonable from a biological point of view. It is interesting to note that when we use the less supported OU-BM model, we find estimates of the heritability to be more in line with the literature, while still on the upper range: 0.35 (95\% HPDI $[0.22,0.51])$ for the GSVL, and 0.21 (95\% HPDI $[0.12,0.32])$ for the CD4 slope. This might raise some concerns on the ability of the MLE model selection procedure to select for the best suited model in this setting. We address both concerns with a simulation study in the next section. 
4.5. Exploration of Model Selection in a Heritability Estimation Context. In the previous section, we estimated the heritability to be lower than $50 \%$. This means that the phylogenetic model (2.2) accounts for at most half of the total variation observed in the dataset, while individual variation (2.3) takes up the remaining part. In the absence of strong phylogenetic signal in the trait, it might be challenging to uncover the true underlying trait evolution model. Using a simulation scheme that is inspired by the empirical dataset, we explore the limits of the MLE model selection procedure in this setting.

\subsubsection{Setting.}

Base Evolutionary Scenario. We used the same fixed HIV tree as in the previous section, normalized so that it had a maximum root to tip height of one. We then simulated a bivariate trait according to a multivariate correlated OU-BM model. By analogy, the two traits are named GSVL and CD4, and the parameters of the process were taken to be similar to the ones inferred in the previous section. For the "GSVL" trait, we took a half-life of $0.3 \%$ of the tree height, a stationary variance $\sigma^{2} /(2 \alpha)$ of 0.1 , and an optimal value (equal to the root conditional value) of 1 . For the "CD4" trait, we took a selection strength of $0(\mathrm{BM})$, a variance of 0.01 , and a root conditional value of -0.1 . The correlation between the two traits was set to -0.9 .

Independent Variations. On top of this evolutionary model, we added independent individual variations at each tip. Under the base scenario, the variance of this extra noise was taken to be equal to the variance of the process ( 0.1 on the GSVL and 0.01 on the CD4). This reflects a heritability of about $50 \%$ (computed to be of $49.4 \%$ for the GSVL and $45.9 \%$ for the CD4). We then multiplied this noise variance by a factor $f$ varying between 0.1 and 3 , leading to a maximal heritability of 0.91 and 0.89 ; and a minimal heritability of 0.25 and 0.22 for both traits, respectively. The estimates for the empirical data set imply a scenario where this factor $f$ is high.

Simulation and Inference. We simulated the OU-BM process using the R package PhylogeneticEM (Bastide, Mariadassou and Robin, 2017; Bastide et al., 2018). Each scenario was repeated 50 times. On each of these datasets, we performed an analysis similar to the previous section, fitting three evolution models (the BM, the true OU-BM and the OU), with independent identically distributed individual variations at each tip.

Questions. We analysed the results to address the two following questions: (i) does the MLE model selection procedure recover the true generative model? and (ii) to what extent is heritability correctly estimated?

\subsubsection{Results.}

Model Selection. The proportion of each model being selected over the 50 repetitions is presented in Table 2. When the individual variation variance is low or equal to the evolutionary variance, the correct OU-BM model is selected in all or most of the cases. When this noise increases however, the proportion drops considerably, with the correct model being selected less than $75 \%$ of the cases when $f=3$.

Estimation of the Heritability. In Figure 5, we show the normalized estimated heritability when we use the correct OU-BM model. When the noise level factor $f$ increases, the variance of the estimates over the 50 repetitions increases substantially, with higher levels of noise leading to an over-estimation of the heritability for both traits. Note that the estimate is more variable for the trait under the OU model (GSVL). This is consistent with the fact that it relies on the estimate of the strength of selection, which is difficult to infer (see Supplementary Figure S7). The empirical coverage level of the $95 \%$ HPD interval remains however relatively high, never dropping below $80 \%$ (see Supplementary Figure S8). 


\begin{tabular}{rrrrrrrr}
\hline Noise Level & 0.1 & 0.25 & 0.5 & 0.75 & 1 & 2 & 3 \\
\hline BM & 0.00 & 0.00 & 0.00 & 0.02 & 0.02 & 0.00 & 0.04 \\
OU-BM & $\mathbf{0 . 9 6}$ & $\mathbf{0 . 9 8}$ & $\mathbf{1 . 0 0}$ & $\mathbf{0 . 8 8}$ & $\mathbf{0 . 9 4}$ & $\mathbf{0 . 7 8}$ & $\mathbf{0 . 6 8}$ \\
OU & 0.04 & 0.02 & 0.00 & 0.10 & 0.04 & 0.22 & 0.28 \\
\hline \multicolumn{7}{c}{ TABLE 2}
\end{tabular}

Proportion of times each model is selected over the 50 replicates. When the independent noise is small compared to the phylogenetic signal, the right model (OU-BM, bold) is almost always selected. When the independent noise become overwhelming, the phylogenetic signal is lost, and the model selection is less efficient. The HIV example explored in Section 4.4 falls under the latter category, with a high noise (or low heritability), which might explain the somewhat unexpected results provided by the model selection in the HIV example.

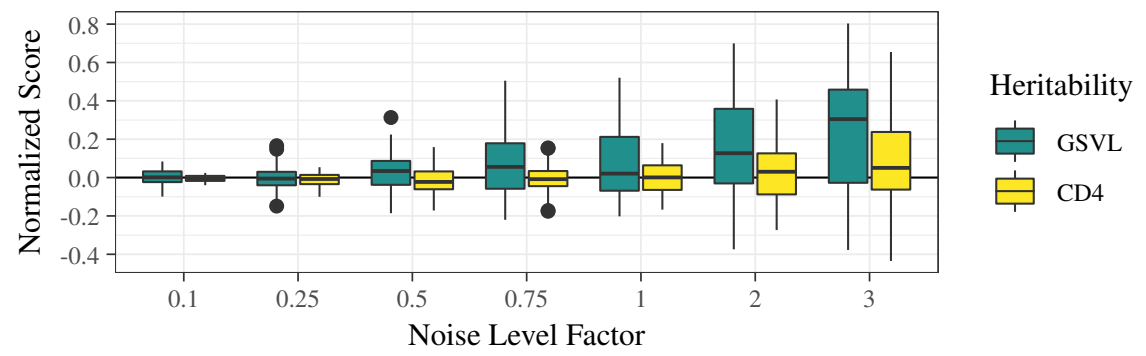

FIG 5. Estimation of the heritability when inferred with the correct (OU-BM) model for various levels of noise. The values are normalized by the true value used in the simulation, so they should converge to 0 (horizontal line). The distribution of the normalized individual estimates over the 50 repetitions are summarized by a box-plot within a violin plot. When the noise level increases, the estimates are more variable, and the heritability tends to be over-estimated.

Selection Strength. When the OU model is incorrectly selected over the OU-BM (which can happen almost one fourth of the time under high levels of noise), then the selection strength on the CD4 trait, that is simulated without selection, is estimated to be generally higher than the selection strength of the GSVL (see Supplementary Figure S7).

Discussion and Caveat. When the noise level is high, we observe three main artifacts in the estimation: (i) the OU model is often wrongly selected over the OU-BM; (ii) the heritability tends to be over-estimated; (iii) when the OU model is selected, the selection strength for the selection-free trait is estimated to be larger than for the trait under selection. These three artifacts are precisely the points that raised questions from a biological point of view in the previous section. This simulation study therefore illustrates that complex models of trait evolution such as the OU, that have recently been advocated in the context of heritability studies (Mitov and Stadler, 2018; Bertels et al., 2018), should be treated with caution when applied to a dataset that is burdened with high levels of noise. In our case, we simulated the data according to the exact same model used for the statistical inference (an OU-BM with residual variance). The performance of the model could be further affected by other sources of variation in a real-world example that are not accounted for in our framework. Examples of such possible mechanisms are discussed below (see Section 5.4).

4.6. Computational Efficiency of the HMC sampler. In all of the applications above, we used the standard HMC sampler described in Section 3.2, that relies on the efficient gradient computation algorithm of Section 2.3. In the case of a simple BM, Hassler et al. (2020) developed a Gibbs sampler on the variance parameter. This approach uses a simplified version of the efficient likelihood computation algorithm described in Section 2.2 to analytically 
integrate missing values. It was shown to be much more efficient than a previous approach based on a numerical integration of these missing values (Cybis et al., 2015), with a minimum 25-fold speed-up in all of the configuration tested (Hassler et al., 2020).

When applying an OU process, such a Gibbs sampler cannot be used anymore, as the joint distribution of the trait values at the tips of the tree cannot be expressed as a simple Kronecker product (see e.g. Clavel, Escarguel and Merceron, 2015, for analytical expressions). Using the transformations described in Appendix $\mathrm{C}$, it is however straightforward to derive an MCMC sampler based on a classical random walk (RW) Metropolis-Hasting algorithm on the space of constrained parameters. Such a sampler still relies on the efficient likelihood computation algorithm of Section 2.2. Contrary to the Gibbs sampler, that can only be used with a set of restricted priors, it has the same flexibility as the HMC sampler and can hence be targeted toward the exact same posterior distribution, allowing for fair comparisons with the HMC.

Given the literature (Neal, 2011; Ji et al., 2020; Fisher et al., 2020), we expect HMC to be more efficient than a simple RW, with better scalability in the number of possibly correlated parameters. To explore computational gains in this context, we reran analyses on the two datasets explored in the previous section. We selected the models with, respectively, the least number of parameters (the BM with no residual error) and the highest number of parameters (the diagonal OU with correlated residual errors). We ran each analysis 10 times and compared the average effective sample size (ESS) per minute for each parameter. We found that HMC delivered appreciable speed-up, with a median 4.37-fold increase in ESS per minute over all the parameters and configuration tested. The speed-up was particularly relevant for the most complex model on the large HIV tree, with an approximate 5 to 10-fold increase in ESS per minute for the variance and selection strength parameters, that are known to be correlated and hence particularly difficult to estimate with a classical RW sampler. We refer to Appendix E.4 for the detailed set up and results of this analysis.

We note that, as both the HMC and RW samplers use the same likelihood computation algorithm (although the RW does not make use of the gradient), the speed-ups reported here are mostly due to the sampling technique, and not the algorithms described in this work. In particular, we here used a classical HMC, with a fixed number of steps and step sizes in the numerical approximation scheme. Further speed-ups may be obtained by using more refined versions of the HMC sampler, for instance using the No-U-Turn Sampler (Hoffman and Gelman, 2014) for optimal exploration of the space, or through the preconditioning of the posterior using an adequate mass matrix (Girolami and Calderhead, 2011; Neal, 2011; Ji et al., 2020). These improvements could be the focus of future work.

\section{Discussion.}

5.1. Fossil Placement Using Continuous Traits. The subject of combining morphological traits with molecular data, which are typically not available for fossils, has received considerable attention over the last few years. Several studies focusing on discrete morphological characters showed that combining both sources of information could improve fossil placement (Wiens, 2009; Wiens et al., 2010), either using a fixed tree through maximum likelihood (Berger and Stamatakis, 2010), or with a total evidence approach (Ronquist et al., 2012; Gavryushkina et al., 2017). A recent trend appears to favor continuous morphological characters over discretized ones, but these may be more challenging to use in an inference framework (Parins-Fukuchi, 2018a). This motivated the development of several methods for fossil placement (Revell et al., 2015; Parins-Fukuchi, 2018b) or divergence time estimation (Álvarez-Carretero et al., 2019) on known phylogenetic trees, using quantitative traits. Given the potentially highly informative value of continuous morphological characters, some attempts have also been made to infer phylogenies without any molecular data, although with 
limited success (Varón-González, Whelan and Klingenberg, 2020). This is consistent with previous findings that sequence information generally tends to dominate over trait information in a joint inference framework (Baele et al., 2017).

In Section 4.3, we showed how the total evidence framework could be used to inform fossil placement. Overall, we observed limited accuracy gains when using continuous trait information (see Section 4.3.2). However, in this example, only three continuous characters were available, offering limited information. It would be interesting to test this framework on more extensive morphometric datasets that include several dozens of traits (ÁlvarezCarretero et al., 2019), and for which the associated signal might be stronger. Combined with phylogenetic factor analysis (Tolkoff et al., 2018), it might provide a good alternative to fixed tree approaches previously mentioned.

5.2. Heritability of Virulence Estimation. In line with previous work (Leventhal and Bonhoeffer, 2016; Mitov and Stadler, 2018), we found in Sections 4.4 and 4.5 that the value and quality of the heritability estimate was strongly dependent on our ability to, first, select for the right model of evolution and, second, to infer the parameters of this model with sufficient precision. As shown in our simulation study, both of these tasks are however challenging when the level of individual variation is high, i.e. the level of heritability is low. This result may not be surprising from a statistical point of view. Indeed, when the level of individual variation is high, then the evolutionary model on the phylogeny only explains a small proportion of the observed tip variation, and it hence becomes difficult to discriminate the signature of one particular model over another in the observed data.

These results call for extreme caution when interpreting not only the heritability estimates obtained in this PCM framework, but also all the other parameters inferred from the model, such as the correlation between the traits (see Appendix E.2). The quality of the estimates, in particular for the OU model, can depend on many factors, including the shape and size of the phylogenetic tree (Cooper et al., 2016). In line with previous recommendations (see e.g. Pennell et al., 2015; Cooper et al., 2016), we found here that it could be useful to complement the empirical analysis of a dataset with tailored simulation studies, designed to explore the potential limits or blind spots of a given configuration.

In the HIV example presented here, the main conclusion that emerges, irrespective of the exact estimate values and favored models, is that the heritability of these virulence traits appears to be limited. Overall, this indicates that host factors constitute an important contribution to the virulence of HIV infections, and that mechanisms underlying complex hostpathogen interactions remain to be explored in more details (Bartha et al., 2017).

5.3. Comparison with Maximum Likelihood Approaches. Numerous maximum likelihood tools exist to fit and compare complex models of evolution on a fixed tree. Such tools are widely used, usually fast, and have proven useful in many situations. For instance, both case studies cited in this article (Schnitzler et al., 2017; Blanquart et al., 2017) used a maximum likelihood framework for their analyses. In addition, the R (R Core Team, 2019) PCMs ecosystem is well developed, so that there exist many specialized packages that cover a wide range of models with various sets of assumptions (see Section 1.3).

Although usually more computationally demanding, Bayesian methods complement maximum likelihood approaches in a number of ways. In particular, the priors' regularizing effect ensures that the parameters estimates remain biologically reasonable (see Appendix C). This feature proved useful in both our applications, where, in contrast, maximum likelihood methods had difficulties in estimating some of the parameters, with estimates lying on arbitrarily fixed upper or lower bounds, yielding poor biological interpretation. 
The Bayesian framework also allows for the computation of the marginal likelihood of a model, that provides a theoretically consistent way to perform model selection. Some penalised likelihood methods, such as the BIC criterion, can be seen as approximations of this gold standard, although rather coarse (Lebarbier and Mary-Huard, 2006). The GSS estimation used here, although computationally intensive, has been shown on the other hand to be one of the most precise methods in a phylogenetic context (Fourment et al., 2019).

Finally, one further strength of our framework is that it is integrated, and allows for the use of all of the extensive BEAST modeling features. Many R packages, on the other hand, although very useful in some situations, have often been developed independently, and their strengths cannot be combined into one global analysis. This is particularly true when one wishes to use a total evidence approach. Most maximum likelihood methods assume a fixed tree, while our Bayesian framework can combine state-of-the-art phylogenetic reconstruction methods with the complex and realistic continuous trait models described here.

5.4. Modeling Assumptions. As detailed in the introduction (see Section 1.3), the framework described here relies on several simplifying assumptions, that are common to most standard PCMs. Relaxing those assumptions usually comes at the cost of a substantial increase in computational complexity, and requires the development of specific algorithms, that are not covered here.

Several simulation studies have been designed to challenge those standard assumptions. They usually rely on a complex trait simulator, that is tailored to a given biological system, and aimed at producing realistic datasets. For instance, in a macro-evolutionary context, Duchen et al. (2020b) study the impact of asymmetrical inheritance on the ability of classical models to describe the produced patterns of trait distribution among species, and show that they can be flawed in some cases. Similarly, in the field of virology, several epidemic models have already been proposed to test the accuracy of virulence heritability estimations (Leventhal and Bonhoeffer, 2016; Mitov and Stadler, 2018).

5.5. Concluding Remarks. Motivated by the need to accommodate OU processes, we have presented an efficient inference procedure for a broad class of trait evolution models in a Bayesian inference framework. Using two empirical examples and a simulation study, we have demonstrated its applicability to answer a large spectrum of biological questions, in fields ranging from paleontology to virology.

At the core of the inference procedure, the likelihood and now gradient computation algorithms are linear in the number of observations, making them efficient on large trees, and applicable to a broad class of Gaussian evolutionary processes, including but not limited to the popular OU model. This algorithm however has worse than quadratic complexity in the number of latent traits propagated on the tree. On the other hand, using techniques such as phylogenetic factor analysis (Tolkoff et al., 2018), this latent dimension can be reduced to a manageable size. In this work, we made use of efficient gradient computation algorithm in a Bayesian context. Note that this gradient could be more broadly exploited in other settings, such as in a maximum likelihood inference.

All the formulas are written here for the general model of Definition 1. Specific formulas are however only implemented for a sub-set of the possible models, namely OU models with diagonal selection strength, with a constant or time-scaled noise, and with only one observation for each tip. Extensions to more general models will be required in order to study specific datasets and answer relevant biological questions. Although the main core mechanism remains unchanged, some derivations may still be needed to propagate the gradient in such complex models. For instance, dealing with the OU with a general selection strength implies taking the derivative of a matrix exponential with respect to a matrix, which is a notoriously difficult problem, and may require some approximations (Al-Mohy and Higham, 
2010). However, in the quest for ever more complex models, particular care should be taken concerning practical and theoretical identifiability issues, as illustrated by our simulation study.

6. Data and Scripts. All the scripts and data used in this manuscript are publicly available as a GitHub repository: https://github.com/pbastide/HMC_OU.

7. Acknowledgments. $\mathrm{PB}$ conducted this research as a postdoctoral fellow funded by the Fonds Wetenschappelijk Onderzoek (FWO, Belgium). The research leading to these results has received funding from the European Research Council under the European Union's Horizon 2020 research and innovation programme (grant agreement no. 725422ReservoirDOCS). The Artic Network receives funding from the Wellcome Trust through project 206298/Z/17/Z. PL acknowledges support by the Research Foundation - Flanders ('Fonds voor Wetenschappelijk Onderzoek - Vlaanderen', G066215N, G0D5117N and G0B9317N). GB acknowledges support from the Interne Fondsen KU Leuven / Internal Funds KU Leuven under grant agreement C14/18/094, and the Research Foundation - Flanders ('Fonds voor Wetenschappelijk Onderzoek - Vlaanderen', G0E1420N). LSTH was supported by startup funds from Dalhousie University, the Canada Research Chairs program, the NSERC Discovery Grant RGPIN-2018-05447, and the NSERC Discovery Launch Supplement DGECR-2018-00181. MAS acknowledges support from National Institutes of Health grant U19 AI135995 and U01 AI151812. We are grateful to the INRAE MIGALE bioinformatics facility (MIGALE, INRAE, 2020. Migale bioinformatics Facility, doi: 10.15454/1.5572390655343293E12) for providing computing resources. PB thanks Pierre Gloaguen for an enlightening discussion about Fisher's identity. The authors thank Jan Schnitzler for sharing the alignment data to reproduce the Musteloidea analyses, as well as Jeffrey S Morris and two anonymous reviewers for their useful comments that helped improve this manuscript.

\section{SUPPLEMENTARY MATERIAL}

Post and Pre-Order Algorithms. Formal and detailed description of the post and preorder algorithms used to compute the likelihood and its gradient. ().

Gradients and Chain Rules Formulas. Formal derivation of the gradient formulas with respect to natural parameters. ().

Constrained Parameters. Description of the smooth transformations used to map the constrained parameters to an unconstrained space. ().

The Heritability Statistics. Case study of the new population variance phylogenetic heritability on a toy example.

().

Supplementary Figures. Supplementary figures for biological applications and simulations.

().

\section{REFERENCES}

Abeles, P. (2016). Efficient Java Matrix Library v0.30. 
AKAIKE, H. (1974). A new look at the statistical model identification. IEEE Transactions on Automatic Control $19716-723$.

Al-Mohy, A. H. and Higham, N. J. (2010). The complex step approximation to the Fréchet derivative of a matrix function. Numerical Algorithms 53 133-148.

Alizon, S., von Wyl, V., Stadler, T., Kouyos, R. D., Yerly, S., Hirschel, B., Böni, J., Shah, C., Klimkait, T., Furrer, H., Rauch, A., Vernazza, P. L., Bernasconi, E., Battegay, M., BürGisser, P., Telenti, A., GÜNThard, H. F. and BonhoefFer, S. (2010). Phylogenetic approach reveals that virus genotype largely determines HIV set-point viral load. PLoS Pathogens 6.

Álvarez-Carretero, S., Goswami, A., Yang, Z. and Dos Reis, M. (2019). Bayesian Estimation of Species Divergence Times Using Correlated Quantitative Characters. Systematic Biology 1-44.

ARISTIDE, L. and MORLON, H. (2019). Understanding the effect of competition during evolutionary radiations: an integrated model of phenotypic and species diversification. Ecology Letters ele.13385.

Aristide, L., dos Reis, S. F., Machado, A. C., Lima, I., Lopes, R. T. and Perez, S. I. (2016). Brain shape convergence in the adaptive radiation of New World monkeys. Proceedings of the National Academy of Sciences $1132158-2163$.

Baele, G., Lemey, P. and Suchard, M. A. (2016). Genealogical Working Distributions for Bayesian Model Testing with Phylogenetic Uncertainty. Systematic Biology 65 250-264.

Baele, G., Suchard, M. A., Rambaut, A. and Lemey, P. (2017). Emerging Concepts of Data Integration in Pathogen Phylodynamics. Systematic Biology 66 47-65.

Bartha, I., Mclaren, P. J., Brumme, C., Harrigan, R., Telenti, A. and Fellay, J. (2017). Estimating the Respective Contributions of Human and Viral Genetic Variation to HIV Control. PLOS Computational Biology 13 e1005339.

BARTOSZEK, K. (2017). Trait evolution with jumps: illusionary normality. September.

Bartoszek, K., Pienaar, J., Mostad, P., Andersson, S. and Hansen, T. F. (2012). A phylogenetic comparative method for studying multivariate adaptation. Journal of Theoretical Biology 314 204-215.

BartoszeK, K., Glémin, S., KAJ, I. and LascouX, M. (2017). Using the Ornstein-Uhlenbeck process to model the evolution of interacting populations. Journal of Theoretical Biology 429 35-45.

BASTIDE, P. (2017). Shifted stochastic processes evolving on trees: application to models of adaptive evolution on phylogenies., PhD Thesis, Université Paris-Saclay.

Bastide, P., Mariadassou, M. and Robin, S. (2017). Detection of adaptive shifts on phylogenies by using shifted stochastic processes on a tree. Journal of the Royal Statistical Society: Series B (Statistical Methodology) 79 1067-1093.

Bastide, P., Ané, C., Robin, S. and Mariadassou, M. (2018). Inference of Adaptive Shifts for Multivariate Correlated Traits. Systematic Biology 67 662-680.

Berger, S. A. and Stamatakis, A. (2010). Accuracy of morphology-based phylogenetic fossil placement under Maximum Likelihood. In ACS/IEEE International Conference on Computer Systems and Applications AICCSA 2010. IEEE.

Bertels, F., Marzel, A., Leventhal, G., Mitov, V., Fellay, J., Günthard, H. F., Böni, J., Yerly, S., Klimkait, T., Aubert, V., Battegay, M., Rauch, A., Cavassini, M., Calmy, A., Bernasconi, E., Schmid, P., Scherrer, A. U., Müller, V., Bonhoeffer, S., Kouyos, R. and Regoes, R. R. (2018). Dissecting HIV Virulence: Heritability of Setpoint Viral Load, CD4+ T-Cell Decline, and Per-Parasite Pathogenicity. Molecular Biology and Evolution 35 27-37.

Betancourt, M. (2017). A Conceptual Introduction to Hamiltonian Monte Carlo. arXiv e-print 1701.02434.

Blanquart, F., Wymant, C., Cornelissen, M., Gall, A., Bakker, M., Bezemer, D., Hall, M., Hillebregt, M., Ong, S. H., Albert, J., Bannert, N., Fellay, J., Fransen, K., Gourlay, A. J., Grabowski, M. K., Gunsenheimer-Bartmeyer, B., Günthard, H. F., Kivelä, P., Kouyos, R., Laeyendecker, O., Littsola, K., Meyer, L., Porter, K., Ristola, M., van Sighem, A., Vanham, G., Berkhout, B., Kellam, P., Reiss, P. and Fraser, C. (2017). Viral genetic variation accounts for a third of variability in HIV-1 set-point viral load in Europe. PLoS Biology 15 1-26.

Boucher, F. C., Démery, V., Conti, E., Harmon, L. J. and UyedA, J. (2018). A General Model for Estimating Macroevolutionary Landscapes. Systematic Biology 67 304-319.

Cappé, O., Moulines, E. and Rydén, T. (2005). Inference in Hidden Markov Models. Springer Series in Statistics. Springer New York, New York, NY.

Cavalli-Sforza, L. L. and Edwards, A. W. F. (1967). Phylogenetic Analysis: Models and Estimation Procedures. Evolution 21550.

Clavel, J., Aristide, L. and Morlon, H. (2019). A Penalized Likelihood Framework for High-Dimensional Phylogenetic Comparative Methods and an Application to New-World Monkeys Brain Evolution. Systematic Biology 68 93-116.

Clavel, J., Escarguel, G. and Merceron, G. (2015). mvmorph : an $\mathrm{r}$ package for fitting multivariate evolutionary models to morphometric data. Methods in Ecology and Evolution 6 1311-1319. 
Cooper, N., Thomas, G. H., Venditti, C., Meade, A. and Freckleton, R. P. (2016). A cautionary note on the use of Ornstein Uhlenbeck models in macroevolutionary studies. Biological Journal of the Linnean Society 118 64-77.

Cybis, G. B., Sinsheimer, J. S., Bedford, T., Mather, A. E., Lemey, P. and Suchard, M. A. (2015). Assessing phenotypic correlation through the multivariate phylogenetic latent liability model. The Annals of Applied Statistics 9 969-991.

Drummond, A. J., Ho, S. Y. W., Phillips, M. J. and Rambaut, A. (2006). Relaxed Phylogenetics and Dating with Confidence. PLoS Biology 4 e88.

Drury, J., Clavel, J., Manceau, M. and Morlon, H. (2016). Estimating the Effect of Competition on Trait Evolution Using Maximum Likelihood Inference. Systematic Biology 65 700-710.

Drury, J. P., Grether, G. F., Garland, T. and Morlon, H. (2018). An Assessment of Phylogenetic Tools for Analyzing the Interplay Between Interspecific Interactions and Phenotypic Evolution. Systematic Biology 67 413-427.

Duchen, P., Leuenberger, C., Szilágyi, S. M., Harmon, L. J., Eastman, J. M., Schweizer, M. and WegmanN, D. (2017). Inference of Evolutionary Jumps in Large Phylogenies using Lévy Processes. Systematic Biology 00 1-14.

Duchen, P., Hautphenne, S., Lehmann, L. and Salamin, N. (2020a). Linking micro and macroevolution in the presence of migration. Journal of Theoretical Biology 486110087.

Duchen, P., Alfaro, M. L., Rolland, J., Salamin, N. and Silvestro, D. (2020b). On the effect of asymmetrical trait inheritance on models of trait evolution. Systematic Biology.

Dudas, G., Carvalho, L. M., Bedford, T., Tatem, A. J., Baele, G., Faria, N. R., Park, D. J., Ladner, J. T., Arias, A., Asogun, D., Bielejec, F., Caddy, S. L., Cotten, M., D’ Ambrozio, J., Dellicour, S., Di Caro, A., Diclaro, J. W., Duraffour, S., Elmore, M. J., Fakoli, L. S., Faye, O., Gilbert, M. L., Gevao, S. M., Gire, S., Gladden-Young, A., Gnirke, A., GobA, A., Grant, D. S., Haagmans, B. L., Hiscox, J. A., Jah, U., Kugelman, J. R., LiU, D., Lu, J., Malboeuf, C. M., Mate, S., Matthews, D. A., Matranga, C. B., Meredith, L. W., Qu, J., Quick, J., Pas, S. D., Phan, M. V. T., Pollakis, G., Reusken, C. B., Sanchez-Lockhart, M., Schaffner, S. F., Schieffelin, J. S., Sealfon, R. S., Simon-Loriere, E., Smits, S. L., Stoecker, K., Thorne, L., ToBin, E. A., Vandi, M. A., Watson, S. J., West, K., Whitmer, S., Wiley, M. R., Winnicki, S. M., Wohl, S., Wölfel, R., Yozwiak, N. L., Andersen, K. G., Blyden, S. O., Bolay, F., Carroll, M. W., Dahn, B., Diallo, B., Formenty, P., Fraser, C., GaO, G. F., Garry, R. F., Goodfellow, I., Günther, S., Happi, C. T., Holmes, E. C., Kargbo, B., KeÏta, S., Kellam, P., Koopmans, M. P. G., Kuhn, J. H., Loman, N. J., Magassouba, N., Naidoo, D., Nichol, S. T., Nyenswah, T., Palacios, G., Pybus, O. G., Sabeti, P. C., Sall, A., Ströher, U., Wurie, I., Suchard, M. A., LEMEY, P. and RAmbAut, A. (2017). Virus genomes reveal factors that spread and sustained the Ebola epidemic. Nature 544 309-315.

Fan, Y., Wu, R., Chen, M. H., Kuo, L. and Lewis, P. O. (2011). Choosing among partition models in Bayesian phylogenetics. Molecular Biology and Evolution 28 523-532.

Felsenstein, J. (1973). Maximum-likelihood estimation of evolutionary trees from continuous characters. American Journal of Human Genetics 25 471-492.

Felsenstein, J. (1985). Phylogenies and the Comparative Method. The American Naturalist 125 1-15.

FElsenstein, J. (2004). Inferring Phylogenies. Sinauer Associates, Sunderland, Massachusetts.

Fisher, A. A., Ji, X., Lemey, P. and Suchard, M. A. (2020). Relaxed Random Walks at Scale. Systematic Biology 1906.04834 .

FITZJOHN, R. G. (2010). Quantitative traits and diversification. Systematic Biology 59 619-633.

FitzJohn, R. G. (2012). Diversitree: Comparative phylogenetic analyses of diversification in R. Methods in Ecology and Evolution 3 1084-1092.

Fitzjohn, R. G., Maddison, W. P. and Otto, S. P. (2009). Estimating trait-dependent speciation and extinction rates from incompletely resolved phylogenies. Systematic Biology 58 595-611.

Fourment, M., Magee, A. F., Whidden, C., Bilge, A., Matsen, F. A. and Minin, V. N. (2019). 19 Dubious Ways to Compute the Marginal Likelihood of a Phylogenetic Tree Topology. Systematic Biology 0 $1-12$.

FRECKLETON, R. P. (2012). Fast likelihood calculations for comparative analyses. Methods in Ecology and Evolution 3 940-947.

Gavryushina, A., Heath, T. A., Ksepka, D. T., Stadler, T., Welch, D. and Drummond, A. J. (2017). Bayesian Total-Evidence Dating Reveals the Recent Crown Radiation of Penguins. Systematic Biology $6657-73$.

Gill, M. S., Tung Ho, L. S., Baele, G., Lemey, P. and Suchard, M. A. (2016). A Relaxed Directional Random Walk Model for Phylogenetic Trait Evolution. Systematic Biology 66 syw093. 
Girolami, M. and CAlderhead, B. (2011). Riemann manifold Langevin and Hamiltonian Monte Carlo methods. Journal of the Royal Statistical Society: Series B (Statistical Methodology) 73 123-214.

Goldberg, E. E., LAnCASter, L. T. and ReE, R. H. (2011). Phylogenetic Inference of Reciprocal Effects between Geographic Range Evolution and Diversification. Systematic Biology 60 451-465.

Goolsb Y, E. W. (2016). Likelihood-Based Parameter Estimation for High-Dimensional Phylogenetic Comparative Models: Overcoming the Limitations of "Distance-Based" Methods. Systematic Biology 65 852-870.

Goolsby, E. W., BRuggeman, J. and AnÉ, C. (2017). Rphylopars : fast multivariate phylogenetic comparative methods for missing data and within-species variation. Methods in Ecology and Evolution 8 22-27.

Hadfield, J. D. and NAKAGAWA, S. (2010). General quantitative genetic methods for comparative biology: phylogenies, taxonomies and multi-trait models for continuous and categorical characters. Journal of Evolutionary Biology 23 494-508.

HAnsen, T. F. (1997). Stabilizing Selection and the Comparative Analysis of Adaptation. Evolution 511341.

Hansen, T. F. and Martins, E. P. (1996). Translating Between Microevolutionary Process and Macroevolutionary Patterns: The Correlation Structure of Interspecific Data. Evolution 501404.

Harmon, L. J. (2019). Phylogenetic Comparative Methods, Version 1. ed. CC-BY-4.0.

Hasegawa, M., Kishino, H. and Yano, T.-A. (1985). Dating of the human-ape splitting by a molecular clock of mitochondrial DNA. Journal of Molecular Evolution 22 160-174.

Hassler, G., Tolkoff, M. R., Allen, W. L., Ho, L. S. T., Lemey, P. and Suchard, M. A. (2020). Inferring Phenotypic Trait Evolution on Large Trees With Many Incomplete Measurements. Journal of the American Statistical Association 1-39.

Hodcroft, E., Hadfield, J. D., Fearnhill, E., Phillips, A., Dunn, D., O’Shea, S., Pillay, D., Leigh Brown, A. J., Celia, A., David, A., Anton, P., Patricia, C., Hannah, C., David, D., Esther, F., Kholoud, P., David, C., Duncan, C., Duncan, C., Simon, C., Valerie, D., Samuel, D., Anna, M. G., Antony, H., Stéphane, H., Steve, K., Paul, K., Linda, L., Andrew, L. B., Tamyo, M., Nicola, M., Chloe, O., Eleni, N., Deenan, P., Andrew, P., Caroline, S., Erasmus, S., Kate, T., Peter, T., Daniel, W., Ian, W., Hongyi, Z., Mark, Z., Jonathan, A., Sris, A., Jane, A., Abdel, B., David, C., Valerie, D., David, D., Martin, F., Brian, G. C., Richard, G., Mark, G., Phillip, H., Teresa, H., Margaret, J., Stephen, K., Clifford, L., Fabiola, M., Mark, N., Chloe, O., Adrian, P., Andrew, P., Deenan, P., Jillian, P., Frank, P., Caroline, S., Memory, S., Achim, S., Anjum, T. and John, W. (2014). The Contribution of Viral Genotype to Plasma Viral Set-Point in HIV Infection. PLoS Pathogens 10.

Hoffman, M. D. and Gelman, A. (2014). The No-U-Turn Sampler: Adaptively Setting Path Lengths in Hamiltonian Monte Carlo. Journal of Machine Learning Research 15 1593-1623.

Housworth, E. A., Martins, E. P. and LYnCh, M. (2004). The phylogenetic mixed model. The American Naturalist 163 84-96.

Jeffreys, H. (1935). Some Tests of Significance, Treated by the Theory of Probability. Mathematical Proceedings of the Cambridge Philosophical Society 31 203-222.

Jetz, W., Thomas, G. H., Joy, J. B., Hartmann, K. and Mooers, A. O. (2012). The global diversity of birds in space and time. Nature $491444-448$.

Ji, X., Zhang, Z., Holbrook, A., Nishimura, A., Baele, G., Rambaut, A., Lemey, P. and SuCHARD, M. A. (2020). Gradients do grow on trees: a linear-time O(N)-dimensional gradient for statistical phylogenetics. Molecular Biology and Evolution 1905.12146.

Jones, A. T., NGuyen, H. D. and MCLachlan, G. J. (2018). logKDE: log-transformed kernel density estimation. Journal of Open Source Software 3870.

KASs, R. E. and RAfTery, A. E. (1995). Bayes Factors. Journal of the American Statistical Association 90 773-795.

Klopfstein, S. and Spasojevic, T. (2019). Illustrating phylogenetic placement of fossils using RoguePlots: An example from ichneumonid parasitoid wasps (Hymenoptera, Ichneumonidae) and an extensive morphological matrix. PLOS ONE 14 e0212942.

Kostikova, A., Silvestro, D., Pearman, P. B. and Salamin, N. (2016). Bridging Inter- and Intraspecific Trait Evolution with a Hierarchical Bayesian Approach. Systematic Biology 65 417-431.

LANDis, M. J., Schraiber, J. G. and Liang, M. (2013). Phylogenetic analysis using Lévy processes: Finding jumps in the evolution of continuous traits. Systematic Biology 62 193-204.

LARTILlot, N. (2014). A phylogenetic Kalman filter for ancestral trait reconstruction using molecular data. Bioinformatics 30 488-496.

Law, C. J., Slater, G. J. and Mehta, R. S. (2018). Lineage Diversity and Size Disparity in Musteloidea: Testing Patterns of Adaptive Radiation Using Molecular and Fossil-Based Methods. Systematic Biology 67 127-144.

Le Gall, J.-F. (2006). Intégration, Probabilités et Processus Aléatoires Technical Report, Ecole Normale Supérieure, Paris. 
LEBARBIER, E. and MARY-HuARD, T. (2006). Une introduction au critère BIC : fondements théoriques et interprétation. Journal de la Société française de Statistiques 147 39-57.

Lemey, P., Rambaut, A., Welch, J. J. and Suchard, M. A. (2010). Phylogeography takes a relaxed random walk in continuous space and time. Molecular Biology and Evolution 27 1877-1885.

Leonardi, M., Librado, P., Der Sarkissian, C., Schubert, M., Alfarhan, A. H., Alquraishi, S. A., Al-Rasheid, K. A. S., Gamba, C., Willerslev, E. and Orlando, L. (2016). Evolutionary Patterns and Processes: Lessons from Ancient DNA. Systematic Biology 66 syw059.

Leventhal, G. E. and Bonhoeffer, S. (2016). Potential Pitfalls in Estimating Viral Load Heritability. Trends in Microbiology 24 687-698.

LeWAndowski, D., KurowickA, D. and JoE, H. (2009). Generating random correlation matrices based on vines and extended onion method. Journal of Multivariate Analysis 100 1989-2001.

LYNCH, M. (1991). Methods for the Analysis of Comparative Data in Evolutionary Biology. Evolution 45 10651080.

Maddison, W. P., Midford, P. E. and OtTo, S. P. (2007). Estimating a binary character's effect on speciation and extinction. Systematic biology 56 701-710.

Magnus, J. R. and Neudecker, H. (1986). Symmetry, 0-1 matrices and Jacobians: A review. Econometric Theory 2 157-190.

Manceau, M., Lambert, A. and Morlon, H. (2016). A unifying comparative phylogenetic framework including traits coevolving across interacting lineages. Systematic Biology 66 syw115.

Mitov, V., BartoszeK, K. and Stadler, T. (2019). Automatic generation of evolutionary hypotheses using mixed Gaussian phylogenetic models. Proceedings of the National Academy of Sciences 201813823.

Mitov, V. and Stadler, T. (2018). A Practical Guide to Estimating the Heritability of Pathogen Traits. Molecular Biology and Evolution 35 756-772.

Mitov, V., BartoszeK, K., Asimomitis, G. and Stadler, T. (2019). Fast likelihood calculation for multivariate Gaussian phylogenetic models with shifts. Theoretical Population Biology.

Müller, N. F., Rasmussen, D. A. and Stadler, T. (2017). The Structured Coalescent and Its Approximations. Molecular Biology and Evolution 34 2970-2981.

NEAL, R. (2011). MCMC Using Hamiltonian Dynamics. In Handbook of Markov Chain Monte Carlo (S. Brooks, A. Gelman, G. L. Jones and X. L. Meng, eds.) 113-162. CRC Press, New York, NY.

Nuismer, S. L. and HARMON, L. J. (2015). Predicting rates of interspecific interaction from phylogenetic trees. Ecology Letters 18 17-27.

OAKs, J. R., CobB, K. A., Minin, V. N. and LEACHÉ, A. D. (2019). Marginal Likelihoods in Phylogenetics: A Review of Methods and Applications. Systematic Biology 68 681-697.

PAGEL, M. (1999). Inferring the historical patterns of biological evolution. Nature 401 877-884.

PARINS-FuKUCHI, C. (2018a). Use of Continuous Traits Can Improve Morphological Phylogenetics. Systematic Biology 67 328-339.

PARINS-FUKUCHI, C. (2018b). Bayesian placement of fossils on phylogenies using quantitative morphometric data. Evolution 72 1801-1814.

Pennell, M. W. and Harmon, L. J. (2013). An integrative view of phylogenetic comparative methods: connections to population genetics, community ecology, and paleobiology. Annals of the New York Academy of Sciences 1289 90-105.

Pennell, M. W., FitzJohn, R. G., Cornwell, W. K. and Harmon, L. J. (2015). Model Adequacy and the Macroevolution of Angiosperm Functional Traits. The American Naturalist 186 E33-E50.

Pybus, O. G., Suchard, M. A., Lemey, P., Bernardin, F. J., Rambaut, A., Crawford, F. W., Gray, R. R., Arinaminpathy, N., Stramer, S. L., Busch, M. P. and Delwart, E. L. (2012). Unifying the spatial epidemiology and molecular evolution of emerging epidemics. Proceedings of the National Academy of Sciences 109 15066-15071.

RABINER, L. R. (1989). A tutorial on hidden Markov models and selected applications in speech recognition. Proceedings of the IEEE 77 257-286.

Rambaut, A., Drummond, A. J., Xie, D., Baele, G. and Suchard, M. A. (2018). Posterior Summarization in Bayesian Phylogenetics Using Tracer 1.7. Systematic Biology 67 901-904.

RAZ, R. (2003). On the Complexity of Matrix Product. SIAM Journal on Computing $321356-1369$.

Revell, L. J., Mahler, D. L., Reynolds, R. G. and Slater, G. J. (2015). Placing cryptic, recently extinct, or hypothesized taxa into an ultrametric phylogeny using continuous character data: A case study with the lizardAnolis roosevelti. Evolution 69 1027-1035.

Ronquist, F., Klopfstein, S., Vilhelmsen, L., Schulmeister, S., Murray, D. L. and RasnitSYN, A. P. (2012). A Total-Evidence Approach to Dating with Fossils, Applied to the Early Radiation of the Hymenoptera. Systematic Biology 61 973-999. 
Salesa, M. J., Antón, M., Siliceo, G., Pesquero, M. D., Morales, J. and Alcalá, L. (2013). A nonaquatic otter (Mammalia, Carnivora, Mustelidae) from the Late Miocene (Vallesian, MN 10) of La Roma 2 (Alfambra, Teruel, Spain): systematics and functional anatomy. Zoological Journal of the Linnean Society 169 448-482.

Schnitzler, J., Theis, C., Polly, P. D. and Eronen, J. T. (2017). Fossils matter - Understanding modes and rates of trait evolution in Musteloidea (Carnivora). Evolutionary Ecology Research 18 187-200.

StAN, D. T. (2017). Stan Modeling Language: User's Guide and Reference Manual v 2.17.0.

Suchard, M. A., Lemey, P., Baele, G., Ayres, D. L., Drummond, A. J. and Rambaut, A. (2018). Bayesian phylogenetic and phylodynamic data integration using BEAST 1.10. Virus Evolution 4 1-5.

TAVARÉ, S. (1986). Some probabilistic and statistical problems in the analysis of DNA sequences. Lectures on mathematics in the life sciences 17 57-86.

R CORE TEAM (2019). R: A Language and Environment for Statistical Computing.

Tolkoff, M. R., Alfaro, M. E., Baele, G., Lemey, P. and Suchard, M. A. (2018). Phylogenetic Factor Analysis. Systematic Biology 67 384-399.

UYEDA, J. C. and HARMON, L. J. (2014). A Novel Bayesian Method for Inferring and Interpreting the Dynamics of Adaptive Landscapes from Phylogenetic Comparative Data. Systematic Biology 63 902-918.

VArón-GonzÁlez, C., Whelan, S. and Klingenberg, P. (2020). Estimating Phylogenies from Shape and Similar Multidimensional Data: Why It Is Not Reliable. Systematic Biology.

Vrancken, B., Lemey, P., Rambaut, A., Bedford, T., Longdon, B., Günthard, H. F. and SUCHARD, M. A. (2015). Simultaneously estimating evolutionary history and repeated traits phylogenetic signal: Applications to viral and host phenotypic evolution. Methods in Ecology and Evolution 6 67-82.

Wang, L.-G., LAm, T. T.-Y., Xu, S., Dai, Z., Zhou, L., Feng, T., Guo, P., Dunn, C. W., Jones, B. R., Bradley, T., Zhu, H., Guan, Y., Jiang, Y. and YU, G. (2019). Treeio: An R Package for Phylogenetic Tree Input and Output with Richly Annotated and Associated Data. Molecular Biology and Evolution.

WiEns, J. J. (2009). Paleontology, Genomics, and Combined-Data Phylogenetics: Can Molecular Data Improve Phylogeny Estimation for Fossil Taxa? Systematic Biology 58 87-99.

Wiens, J. J., Kuczynski, C. A., Townsend, T., Reeder, T. W., Mulcahy, D. G. and Sites, J. W. (2010). Combining Phylogenomics and Fossils in Higher-Level Squamate Reptile Phylogeny: Molecular Data Change the Placement of Fossil Taxa. Systematic Biology 59 674-688.

XIE, W., Lewis, P. O., FAN, Y., Kuo, L. and Chen, M.-H. (2011). Improving Marginal Likelihood Estimation for Bayesian Phylogenetic Model Selection. Systematic Biology 60 150-160.

YANG, Z. (1994). Maximum likelihood phylogenetic estimation from DNA sequences with variable rates over sites: Approximate methods. Journal of Molecular Evolution 39 306-314.

YU, G., Smith, D. K., ZhU, H., GuAN, Y. and LAM, T. T.-Y. (2017). <scp $>$ ggtree $</$ scp $>$ : an $<$ scp $>$ r $</$ scp $>$ package for visualization and annotation of phylogenetic trees with their covariates and other associated data. Methods in Ecology and Evolution 8 28-36.

YU, G., LAM, T. T.-Y., ZHU, H. and GuAN, Y. (2018). Two Methods for Mapping and Visualizing Associated Data on Phylogeny Using Ggtree. Molecular Biology and Evolution 35 3041-3043. 